Indifference Pricing of Pure Endowments and Life Annuities

Under Stochastic Hazard and Interest Rates

2 October 2006

Michael Ludkovski

Department of Mathematics

University of Michigan

Ann Arbor, Michigan, 48109

mludkov@umich.edu

Virginia R. Young

Department of Mathematics

University of Michigan

Ann Arbor, Michigan, 48109

vryoung@umich.edu 


\title{
Indifference Pricing of Pure Endowments and Life Annuities Under Stochastic Hazard and Interest Rates
}

\begin{abstract}
We study indifference pricing of mortality contingent claims in a fully stochastic model. We assume both stochastic interest rates and stochastic hazard rates governing the population mortality. In this setting we compute the indifference price charged by an insurer that uses exponential utility and sells $k$ contingent claims to $k$ independent but homogeneous individuals. Throughout we focus on the examples of pure endowments and temporary life annuities. We begin with a continuous-time model where we derive the linear pdes satisfied by the indifference prices and carry out extensive comparative statics. In particular, we show that the price-per-risk grows as more contracts are sold. We then also provide a more flexible discrete-time analogue that permits general hazard rate dynamics. In the latter case we construct a simulation-based algorithm for pricing general mortality-contingent claims and illustrate with a numerical example.
\end{abstract}

Keywords: Stochastic mortality, indifference pricing, mortality-contingent claims, exponential utility, nonlinear expectations.

JEL Codes: G11, G13, G22, C44.

\section{Introduction}

We consider the problem of pricing mortality-contingent claims when the underlying rate of mortality is random. The insurance markets where such contracts are traded, are inherently incomplete because insurance events are not generally hedgeable. The randomness in the hazard rate is also non-traded. Moreover, the concept of no-arbitrage is difficult to apply in connection with mortality of individuals.

To overcome these difficulties, we propose to apply indifference pricing via expected utility for the insurance markets. To achieve maximum analytical tractability we use exponential utility. While the assumption of (exponential) utility valuation might not be very realistic, it serves as a good illustration of a nonlinear pricing mechanism. Moreover, thanks to the convenient wealth-invariance property of exponential utility, we can isolate the effect of risk-aversion on the contract price. Indifference pricing is a well-known tool in pricing in incomplete financial markets and was pioneered by the work of Hodges and Neuberger (1989) in pricing European options in the presence of proportional transaction costs and later extended by Davis et al. (1993). Since then, indifference pricing has been applied in many different areas of financial and insurance mathematics, such as credit derivatives (Bielecki and Jeanblanc; 2004), insurance (Young and Zariphopoulou; 2002), 
real options (Henderson; 2005) and energy derivatives (Ludkovski; 2006). We refer the reader to the excellent survey of Henderson and Hobson (2005) for the current state of research.

Indifference pricing is not the only method for pricing in incomplete markets. For example, in related work Blanchet-Scalliet et al. (2005) valued assets that mature at a random time by using the principle of no arbitrage; the resulting pricing rule is, therefore, linear. Alternatively, Milevsky et al. (2005) used the notion of instantaneous Sharpe ratio to compute risk premiums. Imperfect replicating or super-replicating strategies have also been introduced to minimize hedging error in an appropriate sense (Leland, 1985; Schweizer, 2001). Nevertheless, we find that indifference pricing is most natural whenever one wishes to focus on the risk preferences of a given insurer, rather than on market equilibrium.

In addition to the fact that insurance markets are incomplete because one cannot buy and sell instruments that hedge mortality-contingent events, we also assume that the rate at which individuals die, the so-called hazard rate is stochastic. Phenomena such as wars, medical breakthroughs and improved lifestyles combine to affect the hazard rate and human longevity in a fluctuating and unpredictable manner (Cairns et al.; 2004). We are not the first to recognize that mortality rates should be viewed as stochastic. Biffis (2005), Schrager (2006), Dahl (2004), as well as Milevsky and Promislow (2001) and Soininen (1995), used diffusion processes to model the force of mortality. Also, demographers and actuaries, such as Lee and Carter (1992), Olivieri (2001), and DiLorenzo and Sibillo (2003), developed methods for projecting mortality.

We contribute to this literature by developing a new fully stochastic model that extends the indifference pricing approach to mortality-contingent claims. Our model is tractable and prices can be obtained as solutions of quasi-linear partial differential equations. This permits us to carry out extensive static comparisons. One major consequence of stochastic force of mortality is that the price-per-risk increases in the number of contracts, as opposed to classical diversification results. This occurs because the stochastic hazard rate induces additional positive correlation among mortality events and leads to super-additivity of the price.

Standard continuous-time models are a powerful tool but are limited to using diffusion processes and require extra assumptions for analytical tractability. Accordingly, we also propose a more flexible discrete-time version. The flexibility is achieved by giving up some analytical structure. Following the ideas of Musiela and Zariphopoulou (2004) we replace the partial differential equation governing the indifference price with a recursive nonlinear expectation procedure. Our aim is a formulation that allows efficient implementation; to 
this end we describe and illustrate how to numerically solve such a model.

The problem of numerically computing nonlinear expectations is related to the literature on backward stochastic differential equations. This has been a very active area of research, see e.g. Bouchard and Touzi (2004), Pagès et al. (2005) and Ludkovski (2006). Our contribution in this direction is a novel application of these ideas in the context of indifference pricing. We construct a simulation-based algorithm that is robust and lends itself well to future extensions. In particular, we hope to use it in future work to address the more general problem of hedging insurance events.

The remainder of this paper is organized as follows. In Section 2, we introduce the continuous-time framework for indifference pricing of mortality-contingent claims. We show that under exponential utility the indifference price of a pure endowment can be decomposed into the product of a bond price and a quantity solving a linear parabolic partial differential equation. In Section 3, we then prove a number of results showing the dependence of the indifference price on model parameters. In particular, we provide upper and lower bounds, as well as prove super-additivity of the indifference price in the number of contracts sold. Section 4 then introduces a discrete-time framework that is flexible and computationally robust. We show that in this context, the indifference price can be represented in terms of nonlinear expectations, and we give a probabilistic algorithm to compute the latter. Section 4 also provides numerical examples to illustrate our results and to compare the continuous-time and discrete-time frameworks. Finally, Section 5 outlines possibilities for extending our model and concludes the paper.

\section{Indifference Pricing in a Continuous-Time Framework}

In this section, we present a continuous-time framework for indifference pricing of mortality contingent claims.

\subsection{Mortality Model and Financial Market}

We begin with a stochastic model for mortality. We assume that the hazard rate $\lambda$ (or force of mortality) of individuals follows a diffusion process such that if the process begins at $\lambda_{0}>0$, then $\lambda_{t}>0$ for all $t \in[0, T]$. Specifically, we assume that

$$
d \lambda_{t}=\mu\left(\lambda_{t}, t\right) \lambda_{t} d t+\sigma(t) \lambda_{t} d B_{t}^{\lambda}
$$

in which $B^{\lambda}$ is a standard Brownian motion on a probability space $(\Omega, \mathcal{F}, \mathbf{P})$. The volatility $\sigma(t)$ is either identically zero, or it is a continuous function of time $t$ bounded below by a positive constant $\kappa$ on $[0, T]$. The drift $\mu(\lambda, t) \lambda$ is a Hölder continuous function of $\lambda$ and 
$t$ for which there exists $\epsilon>0$ such that if $0<\lambda<\epsilon$, then $\mu(\lambda, t)>0$ for all $t \in[0, T]$. After Lemma 3.2 below, we add additional requirements for $\mu$. Note that if $\sigma \equiv 0$, then $\lambda$ is deterministic, and in this case, we write $\lambda(t)$ to denote the deterministic hazard rate at time $t$. An example of (2.1) is the mean-reverting Brownian Gompertz model (MBRG) of Milevsky and Promislow (2001) that we shall use in the numerical example in Section 4.

Suppose an insurer issues a mortality derivative to an individual, namely a contract whose payments are contingent on the individual being alive, an event in turn contingent on $\lambda$. Two common examples are a pure endowment that pays $\$ 1$ at time $T$ if the individual is alive at that time, and a temporary life annuity that pays $\$ 1$ per unit of time as long as the individual is alive, but at most until $T$. Furthermore, we also consider the situation where the insurer issues $k$ such contracts to $k$ separate individuals, whose lives are conditionally independent given $\lambda$, but whose hazard rates are all governed by the same $\lambda$.

To hedge its exposure, the insurer can invest in the money market and in defaultfree zero-coupon bonds that pay $\$ 1$ at time $T_{1}>T$. In this setting, we do not consider investment in stocks or other risky assets, because the insurance contracts are only interest rate-sensitive and therefore the hedging demand for other assets is zero. To model the relevant interest rates and bond prices we use an Affine Term Structure based on the short rate and the bond market's price of risk (Björk, 1998). The dynamics of the short rate $r$, which is the rate at which the money market increases, are given by

$$
d r_{t}=\left(a_{0}(t) r_{t}+b_{0}(t)\right) d t+\sqrt{c(t) r_{t}+d(t)} d B_{t}
$$

in which $a_{0}, b_{0}, c$, and $d$ are deterministic functions of time, and $B$ is a standard Brownian motion with respect to the probability space $(\Omega, \mathcal{F}, \mathbf{P})$, independent of $B^{\lambda}$. This probability space supports the following filtrations: $\mathcal{F}_{t}^{r}=\sigma\left(r_{s}: 0 \leq s \leq t\right), \mathcal{F}_{t}^{\lambda}=\sigma\left(\lambda_{s}: 0 \leq s \leq t\right)$, $\tilde{\mathcal{F}}_{t}=\mathcal{F}_{t}^{r} \vee \mathcal{F}_{t}^{\lambda}=\sigma\left(\left(B_{s}, B_{s}^{\lambda}\right): 0 \leq s \leq t\right)$.

From Lamberton and Lapeyre (1996, Chapter 6), there exists an $\mathcal{F}^{r}$-adapted process $q$, the so-called bond market price of risk, such that the time- $t$ price of a default-free zero-coupon bond that pays $\$ 1$ at time $T_{1}$ is given by

$$
F\left(r, t ; T_{1}\right)=\mathbf{E}_{\mathbf{Q}}\left[\mathrm{e}^{-\int_{t}^{T_{1}} r_{s} d s} \mid r_{t}=r\right],
$$

in which $\mathbf{Q}$ is the probability measure with Radon-Nikodym derivative with respect to $\mathbf{P}$ given by

$$
\left.\frac{d \mathbf{Q}}{d \mathbf{P}}\right|_{\mathcal{F}_{T_{1}}^{r}}=\mathrm{e}^{-\int_{0}^{T_{1}} q_{s} d B_{s}-\frac{1}{2} \int_{0}^{T_{1}} q_{s}^{2} d s} .
$$


It follows that $B^{Q}$, with $B_{t}^{Q}=B_{t}+\int_{0}^{t} q_{s} d s$, is a standard Brownian motion with respect to $\mathbf{Q}$.

Assume that the bond market's price of risk, $q$, is of the form $q_{t}=q\left(r_{t}, t\right)$, in which

$$
q(r, t)=\frac{\left[a_{0}(t)-a(t)\right] r+\left[b_{0}(t)-b(t)\right]}{\sqrt{c(t) r+d(t)}},
$$

for some deterministic functions $a$ and $b$. It follows that with respect to the risk-neutral measure $\mathbf{Q}$, the short rate $r$ follows the dynamics

$$
d r_{t}=\left(a(t) r_{t}+b(t)\right) d t+\sqrt{c(t) r_{t}+d(t)} d B_{t}^{Q} .
$$

Note that the short rate process has the same form of dynamics under the risk-neutral and physical measures.

In addition to the money market, the insurer can purchase zero-coupon bonds that pay $\$ 1$ at time $T_{1}>T$, in which $T$ is the maturity date of the derivative. From Björk (1998), we know that the $T_{1}$-bond price $F$ from (2.3) solves the following partial differential equation (pde):

$$
F_{t}+(a(t) r+b(t)) F_{r}+\frac{1}{2}(c(t) r+d(t)) F_{r r}-r F=0, \quad F\left(r, T_{1} ; T_{1}\right)=1,
$$

Alternatively, $F$ is given by

$$
\left\{\begin{array}{l}
F\left(r, t ; T_{1}\right)=\mathrm{e}^{A\left(t, T_{1}\right)-C\left(t, T_{1}\right) r} \\
C_{t}+a(t) C-\frac{1}{2} c(t) C^{2}=-1, \quad C\left(T_{1}, T_{1}\right)=0 \\
A_{t}=b(t) C-\frac{1}{2} d(t) C^{2}, \quad A\left(T_{1}, T_{1}\right)=0 .
\end{array}\right.
$$

For brevity, we write from now on, $C \equiv C\left(t, T_{1}\right)$. We can use the pde in $(2.7)$ to obtain the dynamics of the $T_{1}$-bond price $F$ starting from a given $r_{t}=r$, for $t \leq s \leq T_{1}$. Indeed,

$$
\left\{\begin{aligned}
d F_{s} & =F_{s}\left[r_{s}-C\left(\Delta a(s) r_{s}+\Delta b(s)\right)\right] d s-F_{s} C \sqrt{c(s) r_{s}+d(s)} d B_{s}, \\
F_{t} & =F\left(r, t ; T_{1}\right),
\end{aligned}\right.
$$

in which $\Delta a(s)=a_{0}(s)-a(s)$ and $\Delta b(s)=b_{0}(s)-b(s)$.

The insurer starts out, at time $t \geq 0$, with an initial endowment of wealth $w \geq 0$, and then proceeds to trade dynamically among the money market and the $T_{1}$-bond. Let $\pi_{s}$ denote the amount invested in the $T_{1}$-bond at time $s$, with the remainder of wealth in the money market account. Thus, the wealth of the insurer $W_{s}$ follows the state dynamics

$$
\left\{\begin{aligned}
d W_{s} & =\left[r_{s} W_{s}-C\left(\Delta a(s) r_{s}+\Delta b(s)\right) \pi_{s}\right] d s-C \sqrt{c(s) r_{s}+d(s)} \pi_{s} d B_{s} \\
W_{t} & =w .
\end{aligned}\right.
$$




\subsection{Pricing Mortality-Contingent Claims}

In Sections 2.2.1 and 2.2.2, we present the value function of an insurer who does not issue the mortality-contingent claim $X$ and the value function of an insurer who does write the derivative, respectively. In Section 2.2.3, we then define the indifference price of $X$, which is the main object of interest in this paper. Throughout we focus on the examples of pure endowments and life annuities.

\subsubsection{Expected Utility without the Claim}

Consider an insurer who does not underwrite any claims. Assume that he seeks to maximize his expected utility of terminal wealth under exponential utility. Let $\gamma$ be a positive parameter that measures the absolute risk aversion of the insurer; we expect a typical well-capitalized insurer to have a small $\gamma$, see the discussion in Young (2003). The corresponding value function $V$ is given by

$$
V(w, r, t)=\sup _{\pi \in \mathcal{A}} \mathbf{E}^{w, r, t}\left[-\mathrm{e}^{-\gamma W_{T}}\right]
$$

in which $\mathbf{E}^{w, r, t}$ denotes expectation conditional on $W_{t}=w$ and $r_{t}=r$, and $\mathcal{A}$ is the set of admissible policies that are $\mathcal{F}_{t}^{r}$-progressively measurable and satisfy the integrability condition $\mathbf{P}^{r, t}\left[\int_{0}^{t}\left(c(s) r_{s}+d(s)\right) \pi_{s}^{2} d s<\infty\right]=1$, for all $t \geq 0$. Such an admissible policy ensures that (2.10) has a unique strong solution (Karatzas and Shreve, 1991, Remark 5.8.4).

In general, there is no consensus about the most appropriate utility function for modeling the preferences of decision makers. Thus, the reader is encouraged to view the use of exponential utility (2.11) as an illustrative example that demonstrates main features of indifference pricing while allowing for explicit computations. If the financial contract that we are pricing can be perfectly replicated then indifference pricing with any utility function will give us the no-arbitrage price. However, in that setting exponential utility will also result in the optimal strategy matching the no-arbitrage hedging strategy, which is not true for a general utility function.

Through a standard verification theorem, one can show that the value function $V$ is the unique smooth solution of the following Hamilton-Jacobi-Bellman (HJB) equation:

$$
\left\{\begin{array}{l}
V_{t}+r w V_{w}+\left(a_{0} r+b_{0}\right) V_{r}+\frac{1}{2}(c r+d) V_{r r} \\
\quad+\max _{\pi}\left[-C(\Delta a r+\Delta b) \pi V_{w}+\frac{1}{2} C^{2}(c r+d) \pi^{2} V_{w w}-C(c r+d) \pi V_{w r}\right]=0 \\
V(w, r, T)=-\mathrm{e}^{-\gamma w} .
\end{array}\right.
$$


For ease of reading, we suppress the dependency of $a_{0}, b_{0}, \Delta a, \Delta b, c$, and $d$ on $t$. A straightforward calculation shows that

$$
V(w, r, t)=-\exp \left(-\frac{\gamma}{F(r, t ; T)} w+\psi(r, t)\right)
$$

in which $\psi$ solves the linear pde

$$
\left\{\begin{array}{l}
\psi_{t}+[(a r+b)-C(t, T)(c r+d)] \psi_{r}+\frac{1}{2}(c r+d) \psi_{r r} \\
\quad-\frac{1}{2(c r+d)}[(\Delta a r+\Delta b)+C(t, T)(c r+d)]^{2}=0 \\
\psi(r, T)=0 .
\end{array}\right.
$$

The corresponding optimal investment $\pi^{V}(w, r, t)$ in the $T_{1}$-bond can be obtained in feedback form as the unique maximizer of the Hamiltonian term in (2.12). Indeed, $\pi^{V}$ is given by (note the appearance of both $C\left(t, T_{1}\right)$ and $C(t, T)$ )

$$
\begin{aligned}
\pi^{V}(w, r, t) & =\frac{1}{C\left(t, T_{1}\right)}\left[\frac{\Delta a r+\Delta b}{c r+d} \frac{V_{w}}{V_{w w}}+\frac{V_{w r}}{V_{w w}}\right] \\
& =\frac{F(r, t ; T)}{\gamma C\left(t, T_{1}\right)}\left[\frac{\gamma}{F(r, t ; T)} C(t, T) w-\psi_{r}-C(t, T)-\frac{\Delta a r+\Delta b}{c r+d}\right]
\end{aligned}
$$

\subsubsection{Expected Utility with the Claim}

Suppose the insurer has the opportunity to write a mortality contingent claim. For sake of definiteness, consider a pure endowment that pays $\$ 1$ at time $T$ if a given individual is alive at that time and nothing otherwise. To model the time of death of the individual introduce a random variable $Y_{t}$ that is equal to $Y_{t}=1$ if the individual is alive at time $t$; otherwise $Y_{t}=0$. We assume that $Y_{t}$ is independent of everything else conditional on all available information $\tilde{\mathcal{F}}_{t}$. To include the uncertainty represented by $Y$ the information structure is extended to $\mathcal{F}_{t} \triangleq \tilde{\mathcal{F}}_{t} \vee \sigma\left(Y_{s}: 0 \leq s \leq t\right)$.

Define the value function $U$ of the writer of this pure endowment by

$$
U(w, r, \lambda, t)=\sup _{\pi \in \mathcal{A}} \mathbf{E}^{w, r, \lambda, t}\left[-\mathrm{e}^{-\gamma\left(W_{T}-Y_{T}\right)}\right]
$$

in which $\mathcal{A}$ is now the set of $\mathcal{F}$-measurable admissible policies for the insurer, and we implicitly condition on $Y_{t}=1$. Wealth $W$ follows the process in (2.10) before time $T$.

More generally, we shall consider an insurer selling $k$ claims $X$ to $k$ individuals all coming from the same population. Accordingly, we will assume that the mortality of the $k$ 
individuals is independent given the hazard rate $\lambda$, but the individuals are subject to the same hazard rate. To keep track of the lives, we introduce the counting process $Y_{s}^{(k)}=m$ if there are exactly $m \in\{0,1, \ldots, k\}$ individuals alive at time $s$ out of the group of $k$ individuals alive at time $t \leq s$. In Markov chain terminology, $Y^{(k)}$ is a simple pure death process with rate $\lambda$. Above we simply have $Y_{s}=Y_{s}^{(1)}$.

The general mortality derivative $X$ is characterized as a triple of functions $\left(x_{1}(y, t)\right.$, $\left.x_{2}(y, t), x_{3}(y)\right)$ that represent, respectively, the continuous payments $x_{1}(y, t)$ made to existing lives, the one-time payment $x_{2}(y, t)$ made when a life terminates (e.g. life insurance claims), and the terminal payment $x_{3}(y)$ made to remaining lives at $T$. The corresponding wealth process $W^{X}$ of the insurer follows

$$
\left\{\begin{aligned}
d W_{s}^{X}= & {\left[r_{s} W_{s}^{a}-C\left(\Delta a r_{s}+\Delta b\right) \pi_{s}-x_{1}\left(Y_{s}^{(k)}, s\right)\right] d s } \\
& -C \sqrt{c r_{s}+d} \pi_{s} d B_{s}-x_{2}\left(Y_{s-}^{(k)}, s\right) d Y_{s}^{(k)} \\
W_{t}^{X}= & w
\end{aligned}\right.
$$

and the value function is given by

$$
U^{X}(w, r, \lambda, t)=\sup _{\pi \in \mathcal{A}} \mathbf{E}^{w, r, \lambda, t}\left[-\mathrm{e}^{-\gamma\left(W_{T}^{X}-x_{3}\left(Y_{T}^{(k)}\right)\right)}\right] .
$$

Observe that equations (2.10) and (2.16) give a special case of (2.17) with $k=1$, $x_{1}=x_{2} \equiv 0$, and $x_{3}(y)=y$. For another example see equations (2.35) and (2.36) below, which illustrate (2.17) in the context of temporary life annuities.

Returning to the value function of a single pure endowment, standard stochastic control methods imply that $U$ is the unique smooth solution of the HJB equation

$$
\left\{\begin{array}{l}
U_{t}+r w U_{w}+\left(a_{0} r+b_{0}\right) U_{r}+\frac{1}{2}(c r+d) U_{r r}+\mu \lambda U_{\lambda}+\frac{1}{2} \sigma^{2} \lambda^{2} U_{\lambda \lambda}-\lambda(U-V) \\
\quad+\max _{\pi}\left[-C(\Delta a r+\Delta b) \pi U_{w}+\frac{1}{2} C^{2}(c r+d) \pi^{2} U_{w w}-C(c r+d) \pi U_{w r}\right]=0 \\
U(w, r, \lambda, T)=-\mathrm{e}^{-\gamma(w-1)}
\end{array}\right.
$$

in which $C=C\left(t, T_{1}\right)$ as in (2.12), and we suppress the dependency of the parameters on $\lambda$ and $t$. One can show that

$$
U(w, r, \lambda, t)=V(w, r, t) \phi(\lambda, t)
$$

in which $\phi$ solves the linear pde

$$
\left\{\begin{array}{l}
\phi_{t}+\mu \lambda \phi_{\lambda}+\frac{1}{2} \sigma^{2} \lambda^{2} \phi_{\lambda \lambda}-\lambda(\phi-1)=0, \\
\phi(\lambda, T)=\mathrm{e}^{\gamma} .
\end{array}\right.
$$


Because $\phi$ solves a linear pde, we can represent $\phi$ as an expectation via the FeynmanKac formula (Karatzas and Shreve, 1991). Indeed, by using the pde of $\phi-1$, one can show that

$$
\phi(\lambda, t)=1+\left(\mathrm{e}^{\gamma}-1\right) \mathbf{E}^{\lambda, t}\left[\mathrm{e}^{-\int_{t}^{T} \lambda_{u} d u}\right]=\mathbf{E}^{\lambda, t}\left[\mathrm{e}^{\gamma Y_{T}}\right]
$$

in which $\mathbf{E}^{\lambda, t}\left[\mathrm{e}^{-\int_{t}^{T} \lambda_{u} d u}\right]$ is the conditional probability that an individual will survive until time $T$ given that she is alive at time $t$.

As in equation (2.15), the corresponding optimal investment $\pi^{U}(w, r, t)$ in the $T_{1}$ bond can be obtained in feedback form as the unique maximizer of the Hamiltonian term in (2.18). However, of more importance is the optimal hedging strategy for the pure endowment, which is given by

$$
\pi_{s}^{U}-\pi_{s}^{V}=\frac{C(s, T)}{C\left(s, T_{1}\right)}\left(W_{s}^{*}-\left(W^{0}\right)_{s}^{*}\right)
$$

in which $W^{*}$ is the optimal wealth process for the insurer with the pure endowment, and $\left(W^{0}\right)^{*}$ is the one for the insurer without the pure endowment. Note that if we let $T_{1}$ approach $T$, then the optimal strategy is to place the difference of the two wealths in the $T$-bond. Thus, at time $t$, when the insurer collects the premium (the difference between the two wealth processes at that time), the insurer places all that money into the $T$-bond and leaves it there until time $T$ when the insurer pays the pure endowment to the individual, if the individual is alive. On average, the insurer will experience a net gain with this scheme.

\subsection{Indifference Pricing}

The time- $t$ indifference price, $H=H^{X}(w, r, \lambda, t)$, for a mortality contingent claim $X$ is the price that makes the issuer of the claim indifferent between not selling it and selling it for the price $H$. Therefore, $H$ solves

$$
V(w, r, t)=U^{X}(w+H, r, \lambda, t)
$$

Because we are using an exponential utility function, $H$ is independent of the initial endowment $w$. This can be easily seen from the special multiplicative factoring of $w$ in (2.13). In general, from (2.23) it follows that $H$ solves a nonlinear pde. However, as we will see below, a relationship such as (2.19) often implies that we can make a transformation which reduces the problem to solving linear pdes.

\subsubsection{Pure Endowments}


For the case of a single pure endowment, we deduce from equations (2.13), (2.19), and (2.23) that

$$
H(r, \lambda, t)=\frac{F(r, t ; T)}{\gamma} \ln \phi(\lambda, t)=\frac{F(r, t ; T)}{\gamma} \ln \mathbf{E}^{\lambda, t}\left[\mathrm{e}^{\gamma Y_{T}}\right]
$$

This phenomenon of representing $\exp \left(\frac{\gamma H(r, \lambda, t)}{F(r, t ; T)}\right)$ as a solution of a (quasi)-linear pde will recur. We can gain insight into the risk-premium of the indifference price given in $(2.24)$ by expanding the price about $\gamma=0$, assuming a small value of $\gamma>0$. Such an expansion shows that

$$
\begin{aligned}
H(r, \lambda, t)= & F(r, t ; T) \mathbf{E}^{\lambda, t}\left[\mathrm{e}^{-\int_{t}^{T} \lambda_{u} d u}\right] \\
& +\frac{1}{2} \gamma F(r, t ; T) \mathbf{E}^{\lambda, t}\left[\mathrm{e}^{-\int_{t}^{T} \lambda_{u} d u}\right]\left(1-\mathbf{E}^{\lambda, t}\left[\mathrm{e}^{-\int_{t}^{T} \lambda_{u} d u}\right]\right)+O\left(\gamma^{2}\right) .
\end{aligned}
$$

Thus, up to first-order terms in $\gamma$, the price is similar to a variance premium principle; this result parallels one in Pratt (1964). Note that the variance operator is super-additive for positively-correlated risks. Thus, the result in (2.25) hints that our pricing mechanism will be super-additive because our risks are positively correlated; see Corollary 3.13 below.

We next consider the price of $k$ pure endowment contracts; thus, (2.16) is replaced by

$$
U^{(k)}(w, r, \lambda, t)=\sup _{\pi \in \mathcal{A}} \mathbf{E}^{w, r, \lambda, t}\left[-\mathrm{e}^{-\gamma\left(W_{T}-Y_{T}^{(k)}\right)}\right] ;
$$

recall that $Y_{T}^{(k)}=m$ if there are exactly $m \in\{0,1, \ldots, k\}$ individuals alive at time $T$ out of the group of $k$ individuals alive at time $t$. Parallel to $(2.18), U^{(k)}$ solves the HJB equation

$$
\left\{\begin{array}{l}
U_{t}^{(k)}+r w U_{w}^{(k)}+\left(a_{0} r+b_{0}\right) U_{r}^{(k)}+\frac{1}{2}(c r+d) U_{r r}^{(k)}+\mu \lambda U_{\lambda}^{(k)}+\frac{1}{2} \sigma^{2} \lambda^{2} U_{\lambda \lambda}^{(k)} \\
\quad-k \lambda\left(U^{(k)}-U^{(k-1)}\right) \\
\quad+\max _{\pi}\left[-C(\Delta a r+\Delta b) \pi U_{w}^{(k)}+\frac{1}{2} C^{2}(c r+d) \pi^{2} U_{w w}^{(k)}-C(c r+d) \pi U_{w r}^{(k)}\right]=0, \\
U^{(k)}(w, r, \lambda, T)=-\mathrm{e}^{-\gamma(w-k)}
\end{array}\right.
$$

in which $U^{(0)}=V$. Note that $U^{(1)}=U$ in (2.18). One can show that

$$
U^{(k)}(w, r, \lambda, t)=V(w, r, t) \phi^{(k)}(\lambda, t)
$$

in which $\phi^{(k)}$ solves the linear pde

$$
\left\{\begin{array}{l}
\phi_{t}^{(k)}+\mu \lambda \phi_{\lambda}^{(k)}+\frac{1}{2} \sigma^{2} \lambda^{2} \phi_{\lambda \lambda}^{(k)}-k \lambda\left(\phi^{(k)}-\phi^{(k-1)}\right)=0, \\
\phi^{(k)}(\lambda, T)=\mathrm{e}^{k \gamma}
\end{array}\right.
$$


with $\phi^{(0)} \equiv 1$. The time- $t$ indifference price of $k$ pure endowments, $H^{(k)}=H^{(k)}(w, r, \lambda, t)$ solves

$$
V(w, r, t)=U^{(k)}\left(w+H^{(k)}, r, \lambda, t\right) .
$$

The solution $H$ of $(2.23)$ is $H^{(1)}$, in the notation of (2.30). Parallel to $(2.24), H^{(k)}$ is independent of wealth and is given by

$$
H^{(k)}(r, \lambda, t)=\frac{F(r, t ; T)}{\gamma} \ln \phi^{(k)}(\lambda, t)
$$

in which $\phi^{(k)}$ solves (2.29). As in the case of a single pure endowment, one can show that the optimal hedging strategy for $k$ pure endowments is to invest the premium in the $T_{1^{-}}$ bond and leave it there until time $T$ when one sells the bond and pays the pure endowment benefits to the survivors.

One can show that $H^{(k)}$ solves the following pde:

$$
\left\{\begin{array}{l}
H_{t}^{(k)}+(a r+b) H_{r}^{(k)}+\frac{1}{2}(c r+d) H_{r r}^{(k)}+\mu \lambda H_{\lambda}^{(k)}+\frac{1}{2} \sigma^{2} \lambda^{2}\left[H_{\lambda \lambda}^{(k)}+\frac{\gamma}{F}\left(H_{\lambda}^{(k)}\right)^{2}\right] \\
\quad-k \lambda \frac{F}{\gamma}\left(1-\exp \left[-\frac{\gamma}{F}\left(H^{(k)}-H^{(k-1)}\right)\right]\right)=r H^{(k)}, \\
H^{(k)}(r, \lambda, T)=k,
\end{array}\right.
$$

in which $F=F(r, t ; T)$. Note that equation $(2.32)$ is reminiscent of the Black-Scholes pricing equation in a complete market, with nonlinear terms that reflect the risk aversion of the insurer.

It is instructive to consider the case for which $\sigma \equiv 0$, that is, the hazard rate $\lambda$ is deterministic. Suppose $\lambda(s)$, for $s \geq t$, is the solution of $d \lambda=\mu(\lambda, s) \lambda d s$ with initial value $\lambda(t)=\lambda ;$ then,

$$
\phi^{(k)}(\lambda, t)=\left[1+\left(\mathrm{e}^{\gamma}-1\right) \mathrm{e}^{-\int_{t}^{T} \lambda(s) d s}\right]^{k},
$$

and the price per risk $\frac{1}{k} H^{(k)}$ is independent of $k$ and equals

$$
\frac{1}{k} H^{(k)}(r, \lambda, t)=\frac{F(r, t ; T)}{\gamma} \ln \mathbf{E}^{\lambda, t}\left[\mathrm{e}^{\gamma Y_{T}^{(1)}}\right] .
$$

Recall that the equality in (2.34) holds for general volatility $\sigma(t)$ when $k=1$; see $(2.21)$. In Section 3, we study properties of the price $H^{(k)}$, or equivalently, of $\phi^{(k)}$.

Remark: The assumption of independence between $r$ and $\lambda$ is crucial for obtaining identities (2.19) and (2.28). If the interest rates and hazard rates were correlated, the pde for 
$\phi^{(k)}$ would be two-dimensional and the problem would be much less tractable. See Section 4.1 below.

\subsubsection{Temporary Life Annuities}

In this section we provide another example of a mortality-contingent claim by pricing temporary life annuities. A temporary life annuity is a financial contract that pays an individual a stream of money until termination time $T$ or until the individual dies, whichever occurs first. In this section, we assume that the life annuity pays at a continuous rate of $\$ 1$ per unit of time. Hence, in the notation of (2.17), this mortality contingent claim is characterized by $x_{1}(y)=y, x_{2}=x_{3} \equiv 0$.

Let $u^{(k)}$ denote the value function of the writer of $k$ life annuities; then $u^{(k)}$ is given by

$$
u^{(k)}(w, r, \lambda, t)=\sup _{\pi \in \mathcal{A}} \mathbf{E}^{w, r, \lambda, t}\left[-\mathrm{e}^{-\gamma W_{T}^{a}}\right]
$$

in which the annuity wealth process $W_{s}^{a}$ follows (compare with (2.17a))

$$
\left\{\begin{aligned}
d W_{s}^{a} & =\left[r_{s} W_{s}^{a}-C\left(\Delta a r_{s}+\Delta b\right) \pi_{s}-Y_{s}^{(k)}\right] d s-C \sqrt{c r_{s}+d} \pi_{s} d B_{s} \\
W_{t}^{a} & =w .
\end{aligned}\right.
$$

Note that $W^{a}$ follows the same process as the wealth in (2.10), except for the continual drain of $Y_{s}^{(k)}$ remaining life annuities, which is represented by the term $-Y_{s}^{(k)} d s$ in (2.36).

Parallel to $(2.27), u^{(k)}$ is the unique, smooth solution of following HJB equation

$$
\left\{\begin{array}{l}
u_{t}^{(k)}+(r w-k) u_{w}^{(k)}+\left(a_{0} r+b_{0}\right) u_{r}^{(k)}+\frac{1}{2}(c r+d) u_{r r}^{(k)}+\mu \lambda u_{\lambda}^{(k)}+\frac{1}{2} \sigma^{2} \lambda^{2} u_{\lambda \lambda}^{(k)} \\
\quad-k \lambda\left(u^{(k)}-u^{(k-1)}\right) \\
\quad+\max _{\pi}\left[-C(\Delta a r+\Delta b) \pi u_{w}^{(k)}+\frac{1}{2} C^{2}(c r+d) \pi^{2} u_{w w}^{(k)}-C(c r+d) \pi u_{w r}^{(k)}\right]=0, \\
u^{(k)}(w, r, \lambda, T)=-\mathrm{e}^{-\gamma w},
\end{array}\right.
$$

in which $u^{(0)}=V$ of $(2.11)$. One can show that

$$
u^{(k)}(w, r, \lambda, t)=V(w, r, t) \rho^{(k)}(r, \lambda, t),
$$

in which $\rho^{(k)}$ solves the quasi-linear pde

$$
\left\{\begin{array}{l}
\rho_{t}^{(k)}+[(a r+b)-C(c r+d)] \rho_{r}^{(k)}+\frac{1}{2}(c r+d)\left(\rho_{r r}^{(k)}-\frac{\left(\rho_{r}^{(k)}\right)^{2}}{\rho_{r r}^{(k)}}\right) \\
\quad+\mu \lambda \rho_{\lambda}^{(k)}+\frac{1}{2} \sigma^{2} \lambda^{2} \rho_{\lambda \lambda}^{(k)}+k \frac{\gamma}{F(r, t ; T)} \rho^{(k)}-k \lambda\left(\rho^{(k)}-\rho^{(k-1)}\right)=0 \\
\rho^{(k)}(r, \lambda, T)=1
\end{array}\right.
$$


with $\rho^{(0)} \equiv 1$. Note that unlike $(2.29), \rho^{(k)}$ depends on both $r$ and $\lambda$.

The total time- $t$ indifference price for the $k$ life annuities, $H^{(k), a}=H^{(k), a}(w, r, \lambda, t)$, solves $V(w, r, t)=u^{(k)}\left(w+H^{(k), a}, r, \lambda, t\right)$. As before, because we are using exponential utility $H^{(k), a}$ is independent of wealth and is given by

$$
H^{(k), a}(r, \lambda, t)=\frac{F(r, t ; T)}{\gamma} \ln \rho^{(k)}(r, \lambda, t)
$$

in which $\rho^{(k)}$ solves $(2.38)$.

As $H^{(k)}$ solves equation $(2.32)$, similarly $H^{(k), a}$ solves the following nonlinear BlackScholes equation:

$$
\left\{\begin{array}{l}
H_{t}^{(k), a}+(a r+b) H_{r}^{(k), a}+\frac{1}{2}(c r+d) H_{r r}^{(k), a} \\
\quad+\mu \lambda H_{\lambda}^{(k), a}+\frac{1}{2} \sigma^{2} \lambda^{2}\left[H_{\lambda \lambda}^{(k), a}+\frac{\gamma}{F}\left(H_{\lambda}^{(k), a}\right)^{2}\right] \\
\quad-k \lambda \frac{F}{\gamma}\left(1-\exp \left[-\frac{\gamma}{F}\left(H^{(k), a}-H^{(k-1), a}\right)\right]\right)=r H^{(k), a}-k \\
H^{(k), a}(r, \lambda, T)=0
\end{array}\right.
$$

in which $F=F(r, t ; T)$. As in equation (2.32), the nonlinear terms reflect the risk aversion of the insurer.

One can show that the corresponding hedging strategy for life annuities is given by

$$
\pi_{s}^{u^{(k)}}-\pi_{s}^{V}=\frac{C(s, T)\left(\left(W^{a}\right)_{s}^{*}-\left(W^{0}\right)_{s}^{*}-H^{(k), a}\left(r_{s}, \lambda_{s}, s\right)\right)}{C\left(s, T_{1}\right)}-\frac{H_{r}^{(k), a}\left(r_{s}, \lambda_{s}, s\right)}{C\left(s, T_{1}\right)}
$$

in which $\left(W^{a}\right)^{*}$ is the optimally controlled wealth process for the insurer of the $k$ life annuities, and $\left(W^{0}\right)^{*}$ is as in equation (2.22). Thus, at time $t$ when the annuities are sold, the amount invested in the $T_{1}$-bond is given by $-\frac{H_{r}^{(k), a}(r, \lambda, t)}{C\left(t, T_{1}\right)}$, as a measure of the sensitivity of the annuity price to changes in the interest rate.

\section{Qualitative Properties of $H^{(k)}$}

In this section, we discuss qualitative properties of the indifference price $H^{(k)}$. For ease of presentation, we focus on the case of $k$ pure endowments, $H^{(k)}=\frac{F}{\gamma} \ln \phi^{(k)}$ rather than on $H^{(k), a}=\frac{F}{\gamma} \ln \rho^{(k)}$, the annuity price. (Note that $\phi^{(k)}$ is independent of $r$, while $\rho^{(k)}$ depends on $r$.) We begin by deriving upper and lower bounds on $H^{(k)}$. We also examine how the price $H^{(k)}$ responds to changes in the model parameters. We then show that $\left\{\frac{1}{k} H^{(k)}: k \geq 1\right\}$ is an increasing sequence, which implies that $H^{(k)}$ is super-additive. 
To this end, we need a comparison principle (Walter, 1970, Section 28). Let $G=$ $\mathbf{R}^{+} \times[0, T]$, and denote by $\mathcal{G}$ the collection of functions on $G$ that are twice-differentiable in their first variable and once-differentiable in their second. We begin by stating a relevant one-sided Lipschitz condition along with growth conditions. We require that a function $g=g(\lambda, t, v, z)$ satisfy the following one-sided Lipschitz condition: For $v>w$,

$$
g(\lambda, t, v, z)-g(\lambda, t, w, q) \leq c(\lambda, t)(v-w)+d(\lambda, t)|z-q|,
$$

with growth conditions on $c$ and $d$ given by

$$
0 \leq c(\lambda, t) \leq K\left(1+(\ln \lambda)^{2}\right), \text { and } 0 \leq d(\lambda, t) \leq K \lambda(1+|\ln \lambda|),
$$

for some constant $K \geq 0$, and for all $(\lambda, t) \in G=\mathbf{R}^{+} \times[0, T]$. Throughout this paper, we rely on the following useful comparison principle, which we obtain from Walter (1970, Section 28).

Theorem 3.1. Define a differential operator $\mathcal{L}$ on $\mathcal{G}$ by

$$
\mathcal{L} v=v_{t}+\frac{1}{2} \sigma^{2}(t) \lambda^{2} v_{\lambda \lambda}+g\left(\lambda, t, v, v_{\lambda}\right)
$$

in which $g$ satisfies (3.1) and (3.2). Suppose $v, w \in \mathcal{G}$ are such that there exists a constant $K \geq 0$ with $v \leq \mathrm{e}^{K(\ln \lambda)^{2}}$ and $w \geq-\mathrm{e}^{K(\ln \lambda)^{2}}$ for large $\lambda$ and for $\lambda$ close to 0 . Then, if $(a)$ $\mathcal{L} v \geq \mathcal{L} w$ on $G$, and if $(b) v(\lambda, T) \leq w(\lambda, T)$ for all $\lambda>0$, then $v \leq w$ on $G$.

Proof. Transform the variables $\lambda$ and $t$ in (3.3) to $y=\ln \lambda$ and $\tau=T-t$, and write $\tilde{v}(y, \tau)=v(\lambda, t)$, etc. Under this transformation, (3.3) becomes

$$
\mathcal{L} \tilde{v}=-\tilde{v}_{\tau}+\frac{1}{2} \tilde{\sigma}^{2}(\tau) \tilde{v}_{y y}+\tilde{h}\left(y, \tau, \tilde{v}, \tilde{v}_{y}\right)
$$

in which $\tilde{h}(y, \tau, \tilde{v}, \tilde{z})=-\frac{1}{2} \tilde{\sigma} 2(\tau) \tilde{z}+\tilde{g}(y, \tau, \tilde{v}, \tilde{z})$, and $\tilde{v}$ is a differential function on $\mathbf{R} \times[0, T]$. Note that $\varphi_{\lambda}=\mathrm{e}^{-y} \tilde{\varphi}_{y}$, so $z=\mathrm{e}^{-y} \tilde{z}$ in going from $g$ to $\tilde{g}$. The differential operator in (3.4) is of the form considered by Walter (1970, pages 213-215); see that reference for the proof of our assertion.

The remaining item to consider is the form of the growth conditions in the original variables $\lambda$ and $t$. From Walter (1970), we know that analogs of (3.1) and (3.2) for $\tilde{h}$ are

$$
\tilde{h}(y, \tau, \tilde{v}, \tilde{z})-\tilde{h}(y, \tau, \tilde{w}, \tilde{q}) \leq \tilde{c}(y, \tau)(\tilde{v}-\tilde{w})+\tilde{d}(y, \tau)|\tilde{z}-\tilde{q}|
$$

with 


$$
0 \leq \tilde{c}(y, \tau) \leq K\left(1+y^{2}\right) \text {, and } 0 \leq \tilde{d}(y, \tau) \leq K(1+|y|)
$$

Under the original variables, the right-hand side of (3.5) becomes $c(\lambda, t)(v-w)+d(\lambda, t) \mid z-$ $q \mid$, in which $c(\lambda, t)=\tilde{c}(y, \tau)$ and $d(\lambda, t)=\tilde{d}(y, \tau) \mathrm{e}^{y}$ because $\tilde{z}=\mathrm{e}^{y} z$. Therefore, $\tilde{d}(y, \tau) \leq$ $K(1+|y|)$ becomes $d(\lambda, t) \leq K \mathrm{e}^{y}(1+|y|)=K \lambda(1+|\ln \lambda|)$.

As a lemma for results to follow, we show that the differential operator associated with $\phi^{(k)}$ satisfies the hypotheses of Theorem 3.1 .

Lemma 3.2. Define $g$, for $k \geq 1$, by

$$
g_{k}(\lambda, t, v, z)=\mu(\lambda, t) \lambda z-k \lambda\left(v-\phi^{(k-1)}\right)
$$

in which $\phi^{(k-1)}$ solves $(2.20)$ with $k$ replaced by $k-1$. Then, $g_{k}$ satisfies the one-sided Lipschitz condition (3.1) on $G$. Furthermore, if $|\mu(\lambda, t)| \leq K(1+|\ln \lambda|)$, then (3.2) holds.

Proof. Suppose $v>w$, then

$$
\begin{aligned}
g_{k}(\lambda, t, v, z)-g_{n}(\lambda, t, w, q) & =\mu(\lambda, t) \lambda(z-q)-n \lambda(v-w) \\
& \leq \lambda|\mu(\lambda, t)| \cdot|z-q| .
\end{aligned}
$$

Thus, (3.1) holds with $c(\lambda, t)=0$ and $d(\lambda, t)=\lambda|\mu(\lambda, t)|$. Note that $d$ satisfies (3.2) if $|\mu(\lambda, t)| \leq K(1+|\ln \lambda|)$.

Assumption 3.3. Henceforth, we assume that the drift $\mu(\lambda, t)$ satisfies the growth condition in the hypothesis of Lemma 3.2. For later purposes (for example, see Theorem 3.7), we also assume that $\mu_{\lambda}$ is Hölder continuous and satisfies the growth condition $\left|\mu_{\lambda}\right| \lambda+|\mu| \leq K\left(1+(\ln \lambda)^{2}\right)$.

In what follows, we apply Theorem 3.1 and Lemma 3.2 repeatedly to determine qualitative properties of the indifference price $H^{(k)}$.

\subsection{Basic Properties of $H^{(k)}$}

In our first application of Theorem 3.1, we show that $H^{(k)} \leq k F$. Note that $F$ is a natural upper bound for the price per risk, $\frac{1}{k} H^{(k)}$, because $F$ is the price of a default-free bond, that is, a bond that pays regardless of whether the individual is alive. We obtain a lower bound in Theorem 3.5 below.

Theorem 3.4. $H^{(k)}(r, \lambda, t) \leq k F(r, t ; T)$ on $\mathbf{R}^{+} \times G$, for $k \geq 0$. 
Proof. From the representation $H^{(k)}=\frac{F}{\gamma} \ln \phi^{(k)}$, it is enough to show that $\phi^{(k)} \leq \mathrm{e}^{k \gamma}$ on $G$. We proceed by induction. Because $\phi^{(0)} \equiv 1, \phi^{(0)} \leq \mathrm{e}^{0 \cdot \gamma}$ is automatic. For $k \geq 1$, assume that $\phi^{(k-1)} \leq \mathrm{e}^{(k-1) \gamma}$, and show that $\phi^{(k)} \leq \mathrm{e}^{k \gamma}$.

Define the differential operator $\mathcal{L}$ on $\mathcal{G}$ by (3.3) with $g=g_{k}$ from (3.7). Because $\phi^{(k)}$ solves (2.29), we have $\mathcal{L} \phi^{(k)}=0$. Note that

$$
\mathcal{L} \mathrm{e}^{k \gamma}=-k \lambda\left(\mathrm{e}^{k \gamma}-\phi^{(k-1)}\right) \leq 0=\mathcal{L} \phi^{(k)} .
$$

Thus, Theorem 3.1 and Lemma 3.2 imply that $\phi^{(k)} \leq \mathrm{e}^{k \gamma}$ on $G$.

Consider the solution $p$ of the following pde on $G$ :

$$
p_{t}+\mu(\lambda, t) \lambda p_{\lambda}+\frac{1}{2} \sigma^{2} \lambda^{2} p_{\lambda \lambda}-\lambda p=0, \quad p(\lambda, T)=1
$$

Note that $p(\lambda, t)$ is the physical probability that an individual alive at time $t$ with hazard rate $\lambda$ at that time survives to time $T$. We can use $p$ to express a lower bound for $H^{(k)}$.

Theorem 3.5. $k F(r, t ; T) p(\lambda, t) \leq H^{(k)}(r, \lambda, t)$ on $\mathbf{R}^{+} \times G$, for any $\gamma>0$.

Proof. It is enough to show that $\phi^{(k)} \geq \mathrm{e}^{k \gamma p}$. We proceed by induction. For $k=0$, the inequality holds with equality because $\phi^{(0)} \equiv 1$. Assume that for $k \geq 1, \phi^{(k-1)} \geq \mathrm{e}^{(k-1) \gamma p}$, and show that $\phi^{(k)} \geq \mathrm{e}^{k \gamma p}$.

Define a differential operator $\mathcal{L}$ on $\mathcal{G}$ by (3.3) with $g=g_{k}$ from (3.7). Because $\phi^{(k)}$ solves (2.29), we have $\mathcal{L} \phi^{(k)}=0$. Also,

$$
\begin{aligned}
\mathcal{L} \mathrm{e}^{k \gamma p} & =k \gamma \mathrm{e}^{k \gamma p}(\lambda p)+\frac{1}{2} \sigma^{2} \lambda^{2} \mathrm{e}^{k \gamma p}\left(k \gamma p_{\lambda}\right)^{2}-k \lambda\left(\mathrm{e}^{k \gamma p}-\phi^{(k-1)}\right) \\
& \geq k \gamma \mathrm{e}^{k \gamma p}(\lambda p)-k \lambda\left(\mathrm{e}^{k \gamma p}-\phi^{(k-1)}\right) \\
& \geq k \gamma \mathrm{e}^{k \gamma p}(\lambda p)-k \lambda\left(\mathrm{e}^{k \gamma p}-\mathrm{e}^{(k-1) \gamma p}\right) \\
& =k \lambda \mathrm{e}^{k \gamma p}\left(\gamma p-1+\mathrm{e}^{-\gamma p}\right),
\end{aligned}
$$

in which the second inequality follows from the induction assumption. The expression in the last line of (3.11) is non-negative because the convex function $\mathrm{e}^{-\gamma p}$ lies above its tangent line at $p=0$, namely $1-\gamma p$.

We have shown that $\mathcal{L} \mathrm{e}^{k \gamma p} \geq 0=\mathcal{L} \phi^{(k)}$. In addition, $\phi^{(k)}(\lambda, T)=\mathrm{e}^{k \gamma}=\mathrm{e}^{k \gamma p(\lambda, T)}$. It follows from Theorem 3.1 and Lemma 3.2 that $\phi^{(k)} \geq \mathrm{e}^{k \gamma p}$ on $G$.

Because $H^{(k)}$ is greater than the price given by $F$ times the expected number of survivors at time $T$ out of the $k$ individuals alive at time $t$, we call $H^{(k)}$ a risk-adjusted 
price, in which $\gamma$ adjusts for the risk aversion of the issuer of the pure endowments (see Theorem 3.8 below for further justification of this).

We next show that $H_{\lambda}^{(k)} \leq 0$. This result is intuitively pleasing because if the current hazard rate $\lambda$ increases, then the probability of surviving until time $T$ decreases, so we expect that the price for pure endowments will decrease. In general, for a claim $F$, if the required payment decreases in $Y^{(k)}$ (as is the case for pure endowments and annuities) then $H_{\lambda}^{(k), F} \leq 0$. We first present a lemma which we use in the proof of Theorem 3.7.

Lemma 3.6. $\phi^{(k)} \geq \phi^{(k-1)}$ on $G$, for $k \geq 1$.

Proof. We proceed by induction. This inequality is true for $k=1$ because $\phi^{(1)}=\phi \geq 1=$ $\phi^{(0)}$ by Theorem 3.4. For $k \geq 2$, assume that $\phi^{(k-1)} \geq \phi^{(k-2)}$, and show that $\phi^{(k)} \geq \phi^{(k-1)}$.

Define a differential operator $\mathcal{L}$ on $\mathcal{G}$ by (3.3) with $g=g_{k}$ from (3.7). Because $\phi^{(k)}$ solves (2.29), we have $\mathcal{L} \phi^{(k)}=0$. Also,

$$
\mathcal{L} \phi^{(k-1)}=(k-1) \lambda\left(\phi^{(k-1)}-\phi^{(k-2)}\right) \geq 0=\mathcal{L} \phi^{(k)} .
$$

In addition, $\phi^{(k)}(\lambda, T)=\mathrm{e}^{k \gamma} \geq \mathrm{e}^{(k-1) \gamma}=\phi^{(k-1)}(\lambda, T)$. Thus, Theorem 3.1 and Lemma 3.2 imply that $\phi^{(k)} \geq \phi^{(k-1)}$ on $G$.

Theorem 3.7. $H_{\lambda}^{(k)} \leq 0$ on $\mathbf{R}^{+} \times G$, for $k \geq 0$.

Proof. It is enough to show that $\phi_{\lambda}^{(k)} \leq 0$ on $G$. We proceed by induction. From $\phi^{(0)} \equiv 1$, it follows that $\phi_{\lambda}^{(0)}=0$. Thus, the inequality holds with equality when $k=0$. For $k \geq 1$, assume that $\phi_{\lambda}^{(k-1)} \leq 0$, and show that $\phi_{\lambda}^{(k)} \leq 0$. First, differentiate $\phi^{(k)}$ 's equation with respect to $\lambda$ to get an equation for $f^{(k)}=\phi_{\lambda}^{(k)}$.

$$
\left\{\begin{array}{l}
f_{t}^{(k)}+\left(\mu_{\lambda} \lambda+\mu-k \lambda\right) f^{(k)}+\left(\mu+\sigma^{2}\right) \lambda f_{\lambda}^{(k)}+\frac{1}{2} \sigma^{2} \lambda^{2} f_{\lambda \lambda}^{(k)} \\
\quad-k\left(\phi^{(k)}-\phi^{(k-1)}\right)+k \lambda f^{(k-1)}=0, \\
f^{(k)}(\lambda, T)=0 .
\end{array}\right.
$$

Define a differential operator $\mathcal{L}$ on $\mathcal{G}$ by (3.3) with $g=g_{k}$ given by

$$
g_{k}(\lambda, t, v, z)=\left(\mu_{\lambda} \lambda+\mu-k \lambda\right) v+\left(\mu+\sigma^{2}\right) \lambda z-k\left(\phi^{(k)}-\phi^{(k-1)}\right)+k \lambda f^{(k-1)} .
$$

It is straightforward to show that, by Assumption 3.3, $g_{k}$ satisfies (3.1) with $c=\left|\mu_{\lambda}\right| \lambda+|\mu|$ and $d=\left(|\mu|+\sigma^{2}\right) \lambda$, in which $c$ and $d$ satisfy the growth conditions in (3.2).

Next, note that because $f^{(k)}=\phi_{\lambda}^{(k)}$ satisfies $(3.13), \mathcal{L} f^{(k)}=0$. Let $\mathbf{0}$ denote the function that is identically 0 . We have $\mathcal{L} \mathbf{0}=-k\left(\phi^{(k)}-\phi^{(k-1)}\right)+k \lambda f^{(k-1)} \leq 0$ by Lemma 
3.6 and by the induction assumption. These observations, together with $f^{(k)}(\lambda, T)=0$, imply that $f^{(k)}=\phi_{\lambda}^{(k)} \leq 0$ on $G$.

\subsection{Comparative Statics for $H^{(k)}$}

Next, we show that as we vary the model parameters, the price $H^{(k)}$ responds consistently with what we expect. First, we show that $H^{(k)}$ increases as $\gamma$ increases. This result justifies the use of the phrase risk parameter when referring to $\gamma$.

Theorem 3.8. Suppose $0<\gamma_{1} \leq \gamma_{2}$, and let $H^{(k), \gamma_{i}}$ be given by (2.31) with $\gamma=\gamma_{i}$ in (2.29), for $i=1,2$ and $n \geq 0$. Then, $H^{(k), \gamma_{1}} \leq H^{(k), \gamma_{2}}$ on $\mathbf{R}^{+} \times G$.

Proof. It is enough to show that $\frac{1}{\gamma_{1}} \ln \phi^{(k), \gamma_{1}} \leq \frac{1}{\gamma_{2}} \ln \phi^{(k), \gamma_{2}}$ on $G$, in which $\phi^{(k), \gamma_{i}}$ has the obvious meaning. Equivalently, we show that $\phi^{(k), \gamma_{1}} \leq\left[\phi^{(k), \gamma_{2}}\right]^{\gamma_{1} / \gamma_{2}}$, and we proceed by induction. It is clear that the inequality holds for $k=0$ because $\phi^{(0), \gamma_{i}} \equiv 1$ for $i=1,2$. For $k \geq 1$, assume that $\phi^{(k-1), \gamma_{1}} \leq\left[\phi^{(k-1), \gamma_{2}}\right]^{\gamma_{1} / \gamma_{2}}$, and show that $\phi^{(k), \gamma_{1}} \leq\left[\phi^{(k), \gamma_{2}}\right]^{\gamma_{1} / \gamma_{2}}$.

Define a differential operator $\mathcal{L}$ on $\mathcal{G}$ by (3.3) with $g=g_{k}$ from (3.7) with $\gamma=\gamma_{1}$. Because $\phi^{(k), \gamma_{1}}$ solves (2.29) with $\gamma=\gamma_{1}$, we have $\mathcal{L} \phi^{(k), \gamma_{1}}=0$. Also,

$$
\begin{aligned}
\mathcal{L} & {\left[\phi^{(k), \gamma_{2}}\right]^{\gamma_{1} / \gamma_{2}}=\frac{\gamma_{1}}{\gamma_{2}}\left[\phi^{(k), \gamma_{2}}\right]^{\frac{\gamma_{1}}{\gamma_{2}}-1}\left(\phi_{t}^{(k), \gamma_{2}}+\mu \lambda \phi_{\lambda}^{(k), \gamma_{2}}+\frac{1}{2} \sigma^{2} \lambda^{2} \phi_{\lambda \lambda}^{(k), \gamma_{2}}\right) } \\
& +\frac{1}{2} \sigma^{2} \lambda^{2} \frac{\gamma_{1}}{\gamma_{2}}\left(\frac{\gamma_{1}}{\gamma_{2}}-1\right)\left[\phi^{(k), \gamma_{2}}\right]^{\frac{\gamma_{1}}{\gamma_{2}-2}}\left(\phi_{\lambda}^{(k), \gamma_{2}}\right)^{2}-k \lambda\left(\left[\phi^{(k), \gamma_{2}}\right]^{\frac{\gamma_{1}}{\gamma_{2}}}-\phi^{(k-1), \gamma_{1}}\right) \\
\leq & \frac{\gamma_{1}}{\gamma_{2}}\left[\phi^{(k), \gamma_{2}}\right]^{\frac{\gamma_{1}}{\gamma_{2}}-1} k \lambda\left(\phi^{(k), \gamma_{2}}-\phi^{(k-1), \gamma_{2}}\right)-k \lambda\left(\left[\phi^{(k), \gamma_{2}}\right]^{\frac{\gamma_{1}}{\gamma_{2}}}-\phi^{(k-1), \gamma_{1}}\right) \\
\leq & k \lambda \frac{\gamma_{1}}{\gamma_{2}}\left[\phi^{(k), \gamma_{2}}\right]^{\frac{\gamma_{1}}{\gamma_{2}}-1}\left(\phi^{(k), \gamma_{2}}-\phi^{(k-1), \gamma_{2}}\right)-k \lambda\left(\left[\phi^{(k), \gamma_{2}}\right]^{\frac{\gamma_{1}}{\gamma_{2}}}-\left[\phi^{(k-1), \gamma_{2}}\right]^{\frac{\gamma_{1}}{\gamma_{2}}}\right),
\end{aligned}
$$

in which the first inequality follows from $\gamma_{1} / \gamma_{2} \leq 1$ and the second follows from the induction assumption. Let $p=1-\gamma_{1} / \gamma_{2} \in[0,1)$, and let $x=\phi^{(k)} / \phi^{(k-1)}-1 \geq 0$. With these assignments, note that the expression in the last line of (3.15) is non-positive if $(1+x)^{p} \leq 1+p x$, which is true because $f(x)=(1+x)^{p}$ is concave and lies below its tangent line at $x=0$, namely $1+p x$.

We have shown that $\mathcal{L}\left[\phi^{(k), \gamma_{2}}\right]^{\gamma_{1} / \gamma_{2}} \leq 0=\mathcal{L} \phi^{(k), \gamma_{1}}$. In addition, $\phi^{(k), \gamma_{1}}(\lambda, T)=$ $\mathrm{e}^{k \gamma_{1}}=\left[\mathrm{e}^{k \gamma_{2}}\right]^{\gamma_{1} / \gamma_{2}}=\left[\phi^{(k), \gamma_{2}}(\lambda, T)\right]^{\gamma_{1} / \gamma_{2}}$. It follows from Theorem 3.1 and Lemma 3.2 that $\phi^{(k), \gamma_{1}} \leq\left[\phi^{(k), \gamma_{2}}\right]^{\gamma_{1} / \gamma_{2}}$ on $G$.

Based on general principles, we expect $\lim _{\gamma \rightarrow 0} H^{(k), \gamma}(r, \lambda, t)=k F(r, t ; T) p(\lambda, t)$, but to show that result is beyond the scope of this paper; see Ilhan et al. (2004) and 
Becherer (2003) for related work. Similarly, $\lim _{\gamma \rightarrow \infty} H^{(k), \gamma}(r, \lambda, t)=k F(r, t ; T)$, which is the super-replicating price of $k$ pure endowments. We also conjecture the corresponding results for life annuities, namely $\lim _{\gamma \rightarrow 0} H^{(k), \gamma, a}(r, \lambda, t)=k \int_{t}^{T} F(r, t ; s) p(\lambda, t ; s) d s$, and $\lim _{\gamma \rightarrow \infty} H^{(k), \gamma, a}(r, \lambda, t)=k \int_{t}^{T} F(r, t ; s) d s$. As in $(3.10), p(\lambda, t ; s)$ denotes the physical probability of an individual who is alive at time $t$ surviving to time $s>t$.

Next, we examine how the risk-adjusted price $H^{(k)}$ varies with the drift and volatility of the stochastic hazard rate. We state the following two theorems without proof because their proofs are similar to earlier ones.

Theorem 3.9. Suppose $\mu_{1} \leq \mu_{2}$ on $G$, and let $H^{(k), \mu_{i}}$ be given by (2.31) with $\mu=\mu_{i}$ in (2.29), for $i=1,2$ and $k \geq 0$. Then, $H^{(k), \mu_{1}} \leq H^{(k), \mu_{2}}$ on $\mathbf{R}^{+} \times G$.

From Theorem 3.9, we learn that as the drift of the hazard rate increases, then the price of a pure endowment decreases. This occurs for essentially the same reason that the price decreases with the hazard rate; see Theorem 3.7.

Theorem 3.10. Suppose $0 \leq \sigma_{1}(t) \leq \sigma_{2}(t)$ on $[0, T]$, and let $H^{(k), \sigma_{i}}$ be given by $(2.31)$ with $\sigma=\sigma_{i}$ in (2.29), for $i=1,2$ and $k \geq 0$. If for $i=1$ or 2 , we have that $H_{\lambda \lambda}^{(k), \sigma_{i}} \geq 0$ for all $k \geq 0$, then $H^{(k), \sigma_{1}} \leq H^{(k), \sigma_{2}}$ on $\mathbf{R}^{+} \times G$.

From Theorem 3.10, we see that if $H^{(k)}$ is convex with respect to $\lambda$, then the riskadjusted price increases if the volatility of the stochastic hazard rate increases.

\subsection{Super-additivity of $H^{(k)}$}

We next show that $H^{(k)}$ is super-additive. Specifically, we show that for $m, n$ nonnegative integers, the following inequality holds:

$$
H^{(m+n)} \geq H^{(m)}+H^{(n)} .
$$

Super-additivity is an expected property of indifference pricing in our setting because as Pratt (1964) demonstrates, the price for a (small) risk $X$ given by expected utility indifference is approximately $E[X]+\alpha \operatorname{Var}(X)$, in which $\alpha>0$ is some parameter related to the risk aversion of the insurer. Therefore, if we use $E[X]+\alpha \operatorname{Var}(X)$ to price two positively correlated risks, then the price for the two risks will be greater than the sum of the prices for the risks individually. In our case, the mortality of the individuals holding the pure endowment is independent given the hazard rate, but the stochastic nature of the latter induces some further "second-order" dependence. It is this dependence that makes the combined risk more risky than the sum of its components (recall that if $\sigma_{\lambda} \equiv 0$ then $H^{(k)}$ is linear in $k$ ). General study of effect of correlation on indifference prices was done 
by Henderson (2002) and Musiela and Zariphopoulou (2004). Also, a related "volumescaling" result for the indifference price of $k X$ where $X$ is a single-event contingent claim was proven by Becherer (2003, Corollary 3.3) in a general semi-martingale incomplete market.

We first study the (limiting) behavior of the price per risk, $\frac{1}{k} H^{(k)}$. We show that the price per risk increases with $k$, which gives super-additivity of $H^{(k)}$ as a corollary. Then, because the price per risk is bounded from above by $F$, we can assert that it has a limit as $k$ goes to infinity.

Theorem 3.11. $\frac{1}{k} H^{(k)}$ increases with respect to $k \geq 1$ on $\mathbf{R}^{+} \times G$.

Proof. It is enough to show that $\phi^{(k)} \geq\left[\phi^{(k-1)}\right]^{k /(k-1)}$ for $k \geq 2$. We proceed by induction and first show that $\phi^{(2)} \geq\left[\phi^{(1)}\right]^{2}$. To that end, define a differential operator $\mathcal{L}$ on $\mathcal{G}$ by (3.3) with $g=g_{2}$ from (3.7). Because $\phi^{(2)}$ solves (2.29) with $k=2$, we have $\mathcal{L} \phi^{(2)}=0$. Also,

$$
\mathcal{L}\left[\phi^{(1)}\right]^{2}=\sigma^{2} \lambda^{2}\left(\phi_{\lambda}^{(1)}\right)^{2} \geq 0=\mathcal{L} \phi^{(2)}
$$

In addition, $\phi^{(2)}(\lambda, T)=\mathrm{e}^{2 \gamma}=\left[\phi^{(1)}(\lambda, T)\right]^{2}$. It follows from Theorem 3.1 and Lemma 3.2 that $\phi^{(2)} \geq\left[\phi^{(1)}\right]^{2}$ on $G$.

For some $k \geq 3$, assume that $\phi^{(k-1)} \geq\left[\phi^{(k-2)}\right]^{(k-1) /(k-2)}$, and show that $\phi^{(k)} \geq$ $\left[\phi^{(k-1)}\right]^{k /(k-1)}$. Define a differential operator $\mathcal{D}$ on $\mathcal{G}$ by $(3.3)$ with $g=g_{k}$ from (3.7). Because $\phi^{(k)}$ solves $(2.29)$, we have $\mathcal{D} \phi^{(k)}=0$. Also,

$$
\begin{aligned}
\mathcal{D}\left[\phi^{(k-1)}\right]^{k /(k-1)}= & k \lambda\left[\phi^{(k-1)}\right]^{\frac{1}{k-1}}\left(\left[\phi^{(k-1)}\right]^{(k-2) /(k-1)}-\phi^{(k-2)}\right) \\
& +\frac{1}{2} \sigma^{2} \lambda^{2} \frac{k}{(k-1)^{2}}\left(\phi_{\lambda}^{(k-1)}\right)^{2}\left[\phi^{(k-1)}\right]^{-\frac{k-2}{k-1}} \\
\geq 0 & =\mathcal{D} \phi^{(k)} .
\end{aligned}
$$

In addition, $\phi^{(k)}(\lambda, T)=\mathrm{e}^{k \gamma}=\left[\phi^{(k-1)}(\lambda, T)\right]^{k /(k-1)}$. It follows from Theorem 3.1 and Lemma 3.2 that $\phi^{(k)} \geq\left[\phi^{(k-1)}\right]^{k /(k-1)}$ on $G$.

By combining Theorems 3.4 and 3.11, we obtain

Corollary 3.12. $\lim _{k \rightarrow \infty} \frac{1}{k} H^{(k)}$ exists and is bounded above by $F$, the price of a defaultfree T-bond. 
Theorem 3.11 also implies the super-additivity of $H^{(k)}$. Indeed, by Theorem 3.11, for any $m, n, \frac{m}{m+n} H^{(m+n)} \geq H^{(m)}$ and $\frac{n}{m+n} H^{(m+n)} \geq H^{(n)}$. By adding the two latter expressions, we obtain

Corollary 3.13. If $m$ and $n$ are non-negative integers, then $H^{(m+n)} \geq H^{(m)}+H^{(n)}$ on $\mathbf{R}^{+} \times G$.

\section{Indifference Pricing in a Discrete-Time Framework}

As a counterpart to the continuous-time model discussed so far, we also consider a discrete-time version. Namely, we continue to assume that the short rate follows (2.2); however, we replace the diffusion for the hazard rate given in (2.1) by a discrete-time model. Let $\mathcal{S}^{\Delta}=\{0, \Delta t, 2 \Delta t, \ldots\}$. We postulate that the information structure of $\lambda$ is discrete and that hazard rate updates are only received at times $t_{m} \triangleq m \Delta t \in \mathcal{S}^{\Delta}$. We allow for general Markovian dynamics of $\lambda_{t}$ over $\mathcal{S}^{\Delta}$ so that $\lambda_{(k+1) \Delta t} \sim \mathbf{P}_{\lambda}\left(\cdot \mid \mathcal{F}_{(k+1) \Delta t}^{r}, \lambda_{k \Delta t}\right)$ for some pre-specified conditional probability measure $\mathbf{P}_{\lambda}$. Hence the new hazard rate depends on its previous value, as well as on the entire history of $r$ (or more generally the latest available financial information). To keep the model tractable, we study the case for which

$$
\lambda_{(m+1) \Delta t} \sim \mathbf{P}_{\lambda}\left(\cdot \mid r_{(m+1) \Delta t}, r_{m \Delta t}, \lambda_{m \Delta t}\right)
$$

Between the update times, we assume that mortality is constant, so that $\lambda_{s}=\lambda_{m \Delta t}$ for $m \Delta t \leq s<(m+1) \Delta t$.

Because the hazard rate $\lambda$ is now piecewise constant, the related stochastic control problem of pricing mortality derivatives is simplified. Between update times, we only face a stochastic interest rate, a variant of a model that was studied by Young and Zariphopoulou (2002). The discrete setting permits generalizations on two counts. First, (4.1) allows nearly arbitrary dependence structure between the mortality rate $\lambda$ and interest rate $r$, as opposed to (2.1)-(2.2), which becomes difficult to study when $r$ and $\lambda$ are correlated. It remains to be seen whether such feature is useful, but in the very least it allows to remove an a priori restriction of the continuous-time model.

Secondly, the discrete setting allows a general Markov process for $\lambda$ without restrictions related to specifying an Itô diffusion. This may be useful empirically as little data is available on mortality dynamics, and it is not clear which continuous-time model to use. In contrast, the discrete time setup can handle, for example, variants of $A R M A(p, q)$ or GARCH-type models that may be easier to calibrate to observed mortality data. One can even incorporate models with jumps in $\lambda$, e.g. to describe pandemics or discovery of new 
cures. Finally, discrete hazard rate dynamics also conform to current actuarial practice which involves infrequent update of $\lambda$ with constant-rate assumption between updates.

Remark: The above setting includes the discrete version of (2.1), i.e. the case when

$$
\lambda_{t+\Delta t}=\lambda_{t}+\mu\left(\lambda_{t}, t\right) \cdot \lambda_{t} \Delta t+\sigma(t) \lambda_{t} \cdot \sqrt{\Delta t} \epsilon_{t}, \quad \epsilon_{t} \sim \mathcal{N}(0,1), \epsilon_{t} \perp \mathcal{F}_{t}^{r} .
$$

Hence, one should view (4.1) as a generalization of discretized dynamics (2.1).

Starting with the dynamics in equations (2.2) and (4.1), we now revisit the problem of finding the indifference price of selling $k$ mortality-contingent claims. As in Sections 2 and 3, we focus on the particular cases of $k$ pure endowments or $k$ temporary life annuities, denoting the corresponding indifference prices as $H^{(k)}(r, \lambda, t)$ and $H^{(k), a}(r, \lambda, t)$ in parallel with (2.31) and (2.40).

\subsection{Pricing Algorithm}

The major tool in our analysis is the following identity about indifference prices of totally unhedgeable risks. A similar result appeared in Becherer (2003, Theorem 4.4).

Lemma 4.1. Let $Z$ be a random variable with distribution $P_{Z}$ such that $Z \perp \mathcal{F}_{T}^{r}$. Given the bond and bank account model of Section 2, consider a payoff of the form $g\left(r_{T}, Z\right)$ for some bounded smooth function $g$. Then, the seller's indifference price (cf. (2.23)) of $g\left(r_{T}, Z\right)$ is

$$
H^{g}(r, t)=\mathbf{E}_{\mathbf{Q}}^{r, t}\left[\frac{1}{\gamma} \ln \left(\int_{\mathbf{R}} \exp \left(\gamma g\left(r_{T}, Z\right)\right) d P_{Z}\right) \cdot \mathrm{e}^{-\int_{0}^{T} r_{s} d s}\right]
$$

Proof. The value function $U(w, r, t)=\sup _{\pi} \mathbf{E}^{w, r, t}\left[-\mathrm{e}^{-\gamma\left(W_{T}-g\left(r_{T}, Z\right)\right)}\right]$ solves an equation analogous to (2.18) with the terminal condition $U(w, r, T)=-\int \exp (-\gamma(w-g(r, z))) d P_{Z}$. Define

$$
\tilde{g}(r) \triangleq \frac{1}{\gamma} \ln \int_{\mathbf{R}} \exp (\gamma g(r, z)) d P_{Z} \equiv \frac{1}{\gamma} \ln E_{Z}[\exp (\gamma g(r, Z))]
$$

Then

$$
U(w, r, T)=-\exp (-\gamma(w-\tilde{g}(r))),
$$

and the equation satisfied by $U(w, r, t)$ is, therefore, identical to the pde one obtains when pricing a payoff $\tilde{g}\left(r_{T}\right)$ in the complete market spanned by the bond and the money market. As shown by Young (2004), the resulting indifference price of $\tilde{g}$ is simply

$$
H^{g}(r, t)=\mathbf{E}_{\mathbf{Q}}^{r, t}\left[\mathrm{e}^{-\int_{t}^{T} r_{s} d s} \tilde{g}\left(r_{T}\right)\right],
$$

which is equivalent to (4.2) once combined with (4.3). 
Next, we extend Lemma 4.1 to the multi-period model consisting of a traded bond and money market account as in Section 2, plus a discrete mortality rate $\lambda$ evolving according to (4.1). The dynamics of (4.1) imply that there exists a (time-dependent) deterministic function $g$ and a probability distribution $P_{Z}$ such that $\lambda_{t+\Delta t} \sim g\left(r_{t+\Delta t}, Z ; r_{t}, \lambda_{t}\right)$ where $Z \perp \mathcal{F}_{t+\Delta t}^{r}$ is an independent random variable with distribution $P_{Z}$. Let $t_{1}=k \Delta t$ and $t_{2}=(k+1) \Delta t$ be two consecutive update times. By the analogy with $(4.2)$, at time $t_{1}$, a payoff $f\left(r_{t_{2}}, \lambda_{t_{2}}\right)$ has the indifference price

$$
H^{f}\left(r_{t_{1}}, \lambda_{t_{1}}, t_{1}\right)=\mathbf{E}_{\mathbf{Q}}\left[\mathrm{e}^{-\int_{t_{1}}^{t_{2}} r_{s} d s} \frac{1}{\gamma} \ln \mathbf{E}\left[\exp \left(\gamma f\left(r_{t_{2}}, \lambda_{t_{2}}\right)\right) \mid \mathcal{F}_{t_{2}}^{r} \vee \mathcal{F}_{t_{1}}^{\lambda}\right] \mid \mathcal{F}_{t_{1}}\right]
$$

Equation (4.5) parallels an expression obtained in Musiela and Zariphopoulou (2004) for a discrete time binomial-tree model. This analogy is an interesting extension that shows that the nonlinear expectation structure is preserved in our non-trivial market setup.

Remark: If instead of (4.5) we use

$$
H^{f, \text { buy }}\left(r_{t_{1}}, \lambda_{t_{1}}, t_{1}\right)=\mathbf{E}_{\mathbf{Q}}\left[-\mathrm{e}^{-\int_{t_{1}}^{t_{2}} r_{s} d s} \frac{1}{\gamma} \ln \mathbf{E}\left[\exp \left(-\gamma f\left(r_{t_{2}}, \lambda_{t_{2}}\right)\right) \mid \mathcal{F}_{t_{2}}^{r} \vee \mathcal{F}_{t_{1}}^{\lambda}\right] \mid \mathcal{F}_{t_{1}}\right],
$$

then we obtain the buyer's indifference price of $f\left(r_{t_{2}}, \lambda_{t_{2}}\right)$.

To be able to price mortality-contingent claims, it remains to understand the structure of the indifference price of a sequence of payments. The crucial result is furnished by the following

Lemma 4.2. Indifference prices are additive in the following sense. Let $C_{1}$ be an obligation with maturity $T_{1}$ (i.e. $C_{1}$ is a bounded $\mathcal{F}_{T_{1}}$-measurable random variable) and $C_{2}$ an obligation with maturity $T_{2}>T_{1}$. Let $H\left(\left[C_{1}, T_{1}\right] ; r, \lambda, t\right)$ be the indifference price of $C_{1}$ at time $t, H\left(\left[C_{2}, T_{2}\right] ; r, \lambda, t\right)$ be the indifference price of $C_{2}$, and $H\left(\left[C_{1}, T_{1} ; C_{2}, T_{2}\right] ; r, \lambda, t\right)$ be the indifference price of receiving both $C_{1}$ and $C_{2}$ at their respective maturity times. Then at time $t<T_{1}$,

$$
H\left(\left[C_{1}, T_{1} ; C_{2}, T_{2}\right] ; r, \lambda, t\right)=H\left(\left[C_{1}, T_{1} ; H\left(\left[C_{2}, T_{2}\right] ; r_{T_{1}}, \lambda_{T_{1}}, T_{1}\right), T_{1}\right] ; r, \lambda, t\right) .
$$

Proof. The lemma says that the indifference price of two obligations is equal to the indifference price of receiving at the earlier time $T_{1}$ the first obligation plus the future indifference price $H\left(\left[C_{2}, T_{2}\right] ; T_{1}, r_{T_{1}}, \lambda_{T_{1}}\right)$ of $C_{2}$. Note that the latter object is an $\mathcal{F}_{T_{1}}$ measurable random variable. In other words, it does not matter if one receives at $T_{1}$ a fixed payment equal to the indifference price of the liability at that time, or the actual liability at $T_{2}$. 
Recall that $H=H\left(\left[C_{2}, T_{2}\right] ; r, \lambda, t\right)$ satisfies

$$
U\left(w+H, r, \lambda, t ;\left[C_{2}, T_{2}\right]\right)=V(w, r, t)
$$

for any time $t<T_{2}$. Here we write $U(\cdot ;[C, T])$ to emphasize the dependence of the value function defined in (2.17) on the claim and maturity date. Combining (4.8) with the dynamic programming principle

$$
U(w, r, \lambda, t ; \cdot)=\sup _{\pi \in \mathcal{A}} \mathbf{E}^{w, r, \lambda, t}\left[U\left(W_{\tau}^{\pi}, r_{\tau}, \lambda_{\tau}, \tau ; \cdot\right)\right]
$$

and the wealth invariance of all the indifference prices concerned, we obtain

$$
\begin{aligned}
U(w, r, \lambda, t ; & {\left.\left[C_{1}, T_{1} ; C_{2}, T_{2}\right]\right)=\sup _{\pi \in \mathcal{A}} \mathbf{E}^{w, r, \lambda, t}\left[U\left(W_{T_{1}}^{\pi}-C_{1}, r_{T_{1}}, \lambda_{T_{1}}, T_{1}+;\left[C_{2}, T_{2}\right]\right)\right] } \\
& =\sup _{\pi \in \mathcal{A}} \mathbf{E}^{w, r, \lambda, t}\left[V\left(W_{T_{1}}^{\pi}-C_{1}-H\left(\left[C_{2}, T_{2}\right], r_{T_{1}}, \lambda_{T_{1}}, T_{1}\right), r_{T_{1}}, T_{1}+\right)\right] \\
& =\sup _{\pi \in \mathcal{A}} \mathbf{E}^{w, r, \lambda, t}\left[U\left(W_{T_{1}}^{\pi}, r_{T_{1}}, \lambda_{T_{1}}, T_{1}-;\left[C_{1}+H\left(\left[C_{2}, T_{2}\right] ; r_{T_{1}}, \lambda_{T_{1}}, T_{1}\right), T_{1}\right]\right)\right] \\
& =U\left(w, r, \lambda, t ;\left[C_{1}+H\left(\left[C_{2}, T_{2}\right] ; r_{T_{1}}, \lambda_{T_{1}}, T_{1}\right), T_{1}\right]\right) .
\end{aligned}
$$

It remains to extend this structure to a multi-period setting. Due to Lemma 4.2 , the indifference price of a claim starting today $H(r, \lambda, t)$ is equal to the expected indifference price of the same claim starting tomorrow plus the current payment (if any). In addition, we must take into account the possibility of one of the lives terminating. The time interval between today and tomorrow is assumed to be sufficiently small to enable us to ignore the possibility of multiple deaths. The latter event risk is yet another (conditionally independent) totally unhedgeable source of uncertainty. As an example, for $k$ temporary life annuities, the future "payoff" at the next update time $t_{2}$ is (cf. (4.4))

$$
f\left(r_{t_{2}}, \lambda_{t_{2}}\right) \simeq\left\{\begin{array}{rll}
H^{(k-1), a}\left(r_{t_{2}}, \lambda_{t_{2}}, t_{2}\right)-(k-1) \Delta t & \text { with prob. } & k \lambda_{t_{1}} \Delta t, \\
H^{(k), a}\left(r_{t_{2}}, \lambda_{t_{2}}, t_{2}\right)-k \Delta t & \text { with prob. } & \left(1-k \lambda_{t_{1}} \Delta t\right) .
\end{array}\right.
$$

For simplicity, we have assumed that payments are only made at times $t_{m} \in \mathcal{S}^{\Delta}$, so that the annuity pays $1 \cdot \Delta t$ dollars to each remaining life at each $t_{m}$.

It follows that the indifference price $H^{(k), a}(r, \lambda, t)$ of a stream of mortality-contingent payments made at times $\Delta t, 2 \Delta t, \ldots$ satisfies the following iteration. Define

$$
\begin{array}{r}
\mathcal{G} H^{(k), a}\left(r_{t_{2}}, \lambda_{t_{2}}, t_{2}\right) \triangleq \mathbf{E}\left[\exp \left(\gamma\left\{H^{(k), a}\left(r_{t_{2}}, \lambda_{t_{2}}, t_{2}\right)-k \Delta t\right\}\right) \cdot\left(1-k \lambda_{t_{1}} \Delta t\right)\right. \\
\left.\quad+\exp \left(\gamma\left\{H^{(k-1), a}\left(r_{t_{2}}, \lambda_{t_{2}}, t_{2}\right)-(k-1) \Delta t\right\}\right) \cdot\left(k \cdot \lambda_{t_{1}} \Delta t\right) \mid \mathcal{F}_{t_{2}}^{r} \vee \mathcal{F}_{t_{1}}^{\lambda}\right]
\end{array}
$$


The $H^{(k), a}$-terms represent the expected future cost taking into account the current hazard rate, and the $k \Delta t$ terms represent the current annuity payment that must be made. Then

$$
H^{(k), a}\left(r, \lambda, t_{1}\right)=\mathbf{E}_{\mathbf{Q}}^{r, \lambda, t_{1}}\left[\mathrm{e}^{-\int_{t_{1}}^{t_{2}} r_{s} d s} \cdot \frac{1}{\gamma} \ln \mathcal{G} H^{(k), a}\left(r_{t_{2}}, \lambda_{t_{2}}, t_{2}\right)\right] .
$$

For pricing a pure endowment, we only need to replace (4.10) with

$$
\begin{gathered}
\mathcal{G} H^{(k)}\left(r_{t_{2}}, \lambda_{t_{2}}, t_{2}\right) \triangleq \mathbf{E}\left[\exp \left(\gamma H^{(k)}\left(r_{t_{2}}, \lambda_{t_{2}}, t_{2}\right)\right) \cdot\left(1-k \cdot \lambda_{t_{1}} \Delta t\right)\right. \\
\left.+\exp \left(\gamma H^{(k-1)}\left(r_{t_{2}}, \lambda_{t_{2}}, t_{2}\right)\right) \cdot\left(k \cdot \lambda_{t_{1}} \Delta t\right) \mid \mathcal{F}_{t_{2}}^{r} \vee \mathcal{F}_{t_{1}}^{\lambda}\right]
\end{gathered}
$$

keeping (4.11) as is. Similar adjustments can be used to price any other mortality-related claim $X$ covering $k$ lives.

Note that above $\lambda$ is evolving under the real-world measure $\mathbf{P}$, while $r$ is evolving under the minimal martingale measure $\mathbf{Q}$ (in (4.11)). This reflects the fact that mortality is nontraded (but partially hedgeable due to possible dependence on interest rates), whereas the interest rate's uncertainty is traded via the bank account and the bond. Also observe that even for pricing a pure endowment in (4.12), the interest rate dependence explicitly enters into the calculations, as opposed to (2.31) where it appeared as simply the bond price $F(r, t ; T)$.

\subsection{Numerical Implementation}

The algorithm defined by equations (4.10) and (4.11) is amenable to numeric computations. Note that all that is necessary is computing conditional expectations, with the crucial one in (4.11). To compute these expectations, one can use either pde methods or probabilistic algorithms. The pde method relies on representing (4.11) as a solution to a linear parabolic pde via the Feynman-Kac formula (Karatzas and Shreve, 1991). Furthermore, the conditional expectation in (4.10) can be computed as a numerical integration of the conditional density $\mathbf{P}_{\lambda}$ of $\lambda_{t+\Delta t}$ given $\left(r_{t+\Delta t}, r_{t}, \lambda_{t}\right)$. Overall, this gives rise to a coupled system of one-dimensional pdes in $r$, parameterized by $\lambda$. We come back to this approach in Section 4.5.

The probabilistic algorithms, on the other hand, are based on Monte-Carlo simulations and treat $r$ and $\lambda$ more equally. Recall that the problem of computing conditional expectations is well known in applied probability and has a natural Monte-Carlo interpretation as a weighted average. Many approaches are possible. Here we propose the projection method, which is a popular choice and is related to the Longstaff and Schwartz (2001) 
scheme for American options. The idea is to view the conditional expectation (4.11) as an $\mathrm{L}^{2}\left(\mathcal{F}_{t}\right)$-functional

$$
\mathbf{E}_{t}:(r, \lambda) \mapsto \mathbf{E}_{\mathbf{Q}}^{r, \lambda, t}\left[\mathrm{e}^{-r_{t} \Delta t} \cdot \frac{1}{\gamma} \ln \mathcal{G} H^{(k), a}\left(r_{t+\Delta t}, \lambda_{t+\Delta t}, t+\Delta t\right)\right]
$$

and to approximate this functional by projecting it onto a truncated set of basis functions $\left\{B_{j}\right\}$,

$$
\mathbf{E}_{t}(r, \lambda) \simeq \sum_{j=1}^{N_{B}} \alpha_{j} B_{j}(r, \lambda),
$$

where $\alpha_{j} \in \mathbf{R}$ are the projection coefficients. The canonical choice is to take $B_{j}=\tilde{B}_{j}, j=$ $1,2, \ldots, N_{B}$, where $\left\{\tilde{B}_{j}\right\}$ is a complete orthonormal family in $\mathrm{L}^{2}(\mathbf{R} \times \mathbf{R})$ (for instance, take $\tilde{B}_{j}(r, \lambda)=\tilde{B}_{j_{1}}^{1}(r) \cdot \tilde{B}_{j_{2}}^{2}(\lambda)$, where $\left\{\tilde{B}_{j_{1}}^{1}\right\}$ and $\left\{\tilde{B}_{j_{2}}^{2}\right\}$ form one-dimensional orthonormal families). In turn, optimal projection is easily approximated by means of standard $\mathrm{L}^{2}$-least squares regression over a set of simulated paths.

To implement the above method, we simulate $N$ paths $\left(r_{m \Delta t}^{n}\right)_{n=1}^{N}$ of $r$ under $\mathbf{Q}$, complemented by $N$ corresponding paths $\left(\lambda_{m \Delta t}^{n}\right)$ of $\lambda$ under $\mathbf{P}$. Suppose that $H^{(k), a}(r, \lambda, t+\Delta t)$ is known for all values of $(r, \lambda)$, and in particular for each pair $\left(r_{t+\Delta t}^{n}, \lambda_{t+\Delta t}^{n}\right)$. Our goal is to apply the iteration in equations (4.10) and (4.11) to obtain $H^{(k), a}(r, \lambda, t)$. First, to compute $\mathcal{G} H^{(k), a}$, simulate $N_{2}$ copies $\left\{\lambda_{t+\Delta t}^{i}\right\}_{i=1}^{N_{2}}$ of $\lambda_{t+\Delta t}$ given $\left(r_{t+\Delta t}, r_{t}, \lambda_{t}\right)$ and any other necessary past information. The conditional expectation of (4.10) is approximated by the average over the simulated values $\mathcal{G} H^{(k), a}\left(r_{t+\Delta t}^{n}, \lambda_{t+\Delta t}^{n}, t+\Delta t\right) \simeq$ $\tilde{\mathcal{G}}_{N_{2}} H^{(k), a}\left(r_{t+\Delta t}^{n}, \lambda_{t+\Delta t}^{n}, t+\Delta t\right)$ with

$$
\begin{gathered}
\tilde{\mathcal{G}}_{N_{2}} H^{(k), a}\left(r_{t+\Delta t}^{n}, \lambda_{t+\Delta t}^{n}, t+\Delta t\right) \triangleq \frac{1}{N_{2}} \sum_{i=1}^{N_{2}} \mathrm{e}^{\gamma\left\{H^{(k), a}\left(r_{t+\Delta t}^{n}, \lambda_{t+\Delta t}^{i}, t+\Delta t\right)-k \Delta t\right\}}\left(1-k \lambda_{t}^{n} \Delta t\right) \\
+\mathrm{e}^{\gamma\left\{H^{(k-1)}\left(r_{t+\Delta t}^{n}, \lambda_{t+\Delta t}^{i}, t+\Delta t\right)-(k-1) \Delta t\right\}} \cdot\left(k \lambda_{t}^{n} \Delta t\right) ;
\end{gathered}
$$

compare with the standard procedure of approximating integrals by Monte-Carlo simulation. Next, to compute $H^{(k), a}\left(r_{t}, \lambda_{t}, t\right)$, regress the $(t+\Delta t)$-values $\mathcal{G} H^{(k), a}\left(r_{t+\Delta t}^{n}, \lambda_{t+\Delta t}^{n}, t+\right.$ $\Delta t$ ) against $\left(r_{t}, \lambda_{t}\right)$. This gives a new (approximate) map $(r, \lambda) \mapsto H^{(k), a}(r, \lambda, t)$. It remains to repeat this procedure backward in time, starting from the expiration date $T=M \Delta t$ with terminal condition $H^{(k), a}(r, \lambda, T)=0$ down to 0 .

\subsection{Summary of Algorithm and Convergence}

The algorithm below computes the indifference price of $K$ copies of a bounded $T$ claim $X$ sold to $K$ separate individuals. The true price $H^{(k), X}(r, \lambda, t), k=0,1, \ldots, K$ is 
approximated by $\varphi^{k}(r, \lambda, t)$. We continue to label $\mathcal{G}$ the nonlinear expectation operator corresponding to $X$, cf. (4.10) and (4.12).

(1) Select a set of basis functions $\left\{B_{j}\right\}$ and algorithm parameters $M, N_{B}, N, N_{2}$.

(2) Generate $N$ paths of the driving process: $\left\{\left(r_{m \Delta t}^{n}, \lambda_{m \Delta t}^{n}\right): m=0,1, \ldots, M, \quad n=\right.$ $1,2, \ldots, N\}$ with fixed initial condition $\left(r_{0}^{n}, \lambda_{0}^{n}\right)=\left(r_{0}, \lambda_{0}\right)$, using (2.2)-(4.1).

(3) Initialize the indifference functions $\varphi^{k}\left(r_{T}^{n}, \lambda_{T}^{n}, T\right)=0$, for $k=0,1, \ldots, K$.

(4) Moving backward in time with $t=m \Delta t, m=M-1, \ldots, 1$ repeat the Loop (5):

(5) For $k=0,1, \ldots, K$, inductively compute:

(5.1) For each pair $\left(r_{t+\Delta t}^{n}, \lambda_{t}^{n}\right)$, simulate $N_{2}$ values of $\lambda_{t+\Delta t}^{i} \sim \mathbf{P}_{\lambda}\left(\lambda_{t+\Delta t} \mid r_{t+\Delta t}^{n}, \lambda_{t}^{n}, r_{t}^{n}\right)$ $i=1,2, \ldots, N_{2}$, and find the future indifference price $\varphi^{k}\left(r_{t+\Delta t}^{n}, \lambda_{t+\Delta t}^{i}, t+\Delta t\right)$.

(5.2) Apply the analogue of (4.14) for claim $X$, storing the result as

$$
g_{n}^{k}=\tilde{\mathcal{G}}_{N_{2}} \varphi^{k}\left(r_{t+\Delta t}^{n}, \lambda_{t+\Delta t}^{n}, t+\Delta t\right) .
$$

(5.3) To evaluate the conditional expectation $\mathbf{E}\left[\frac{1}{\gamma} \ln g_{n}^{k} \mathrm{e}^{-r_{m \Delta t}^{n} \Delta t} \mid \mathcal{F}_{m \Delta t}\right]$ regress $\left\{\mathrm{e}^{-r_{m \Delta t}^{n} \Delta t} \cdot \ln g_{n}^{k}\right\}, n=1,2, \ldots, N$ against the current values of basis functions $\left\{B_{j}\left(r_{m \Delta t}^{n}, \lambda_{m \Delta t}^{n}\right)\right\}_{j=1}^{N_{B}}$.

(5.4) The resulting basis coefficients $\left\{\alpha_{j}\right\}(m \Delta t)$ form the new map

$$
\varphi^{k}(r, \lambda, m \Delta t) \triangleq \sum_{j=1}^{N_{B}} \alpha_{j} B_{j}(r, \lambda) \simeq H^{(k), X}(r, \lambda, m \Delta t) .
$$

(6) End Loop

(7) Output

$$
\varphi^{k}\left(r_{0}, \lambda_{0}, 0\right)=\frac{1}{N} \sum_{n=1}^{N} \varphi^{k}\left(r_{\Delta t}^{n}, \lambda_{\Delta t}^{n}, \Delta t\right) \simeq H^{(k), X}\left(r_{0}, \lambda_{0}, 0\right) .
$$

The above algorithm is closely related to numerical solution of backward stochastic differential equations, see for instance Bouchard and Touzi (2004). In both situations conditional expectations are approximated with $\mathrm{L}^{2}$-projections on finite bases. Convergence analysis is intricate because the current approximation $\varphi^{k}(\cdot ; m \Delta t)$ depends on the earlier maps $\varphi^{k-1}(\cdot ; m \Delta t)$ and $\varphi^{k}(\cdot ;(m+1) \Delta t)$ which themselves were approximated. As a result, the errors in computing $\varphi^{k}$ are propagated backwards in time. In addition, we have the nonlinear map $\mathcal{G}$ which is applied in conjunction with conditional expectations.

Nevertheless, a quite precise analysis may be carried out, following similar work in Gobet et al. (2006) and Carmona and Ludkovski (2005). The key tool are a priori estimates 
on the true solutions $H^{(k), X}$ which allow to introduce truncation functions and control the projection error and the nonlinearity of $\mathcal{G}$. Let

$$
\operatorname{PrErr}{ }^{k}(r, \lambda, t)=\inf _{\alpha_{1}, \ldots, \alpha_{N_{B}}} \mathbf{E}^{r, \lambda, 0}\left\|H^{(k), X}\left(r_{t}, \lambda_{t}, t\right)-\sum_{j=1}^{N_{B}} \alpha_{j} B_{j}\left(r_{t}, \lambda_{t}\right)\right\|^{2},
$$

denote the expected squared projection error and

$$
\begin{aligned}
\operatorname{AvErr}^{k}(r, \lambda, t)= & \mathbf{E}^{r, \lambda, t} \| \mathcal{G} H^{(k), X}\left(r_{t+\Delta t}, \lambda_{t+\Delta t}, t+\Delta t\right) \\
& -\tilde{\mathcal{G}}_{N_{2}} H^{(k), X}\left(r_{t+\Delta t}^{n}, \lambda_{t+\Delta t}^{i}, t+\Delta t\right) \|^{2}
\end{aligned}
$$

denote the expected squared averaging error. The following proposition summarizes the convergence estimate. We omit the proof which closely follows the proof of Theorem 2 in Gobet et al. (2006).

Theorem 4.1. Fix a complete orthonormal family $\left\{B_{j}\right\}_{j=1}^{\infty}$. Then there exists a constant $C$ depending on the claim $X$ and the parameters of (2.2)-(4.1) such that (recall $M=\frac{1}{\Delta t}$ )

$$
\begin{aligned}
\max _{0 \leq m \leq M} \mathbf{E} & {\left[\frac{1}{N} \sum_{n=1}^{N}\left|H^{(k), X}\left(r_{m \Delta t}^{n}, \lambda_{m \Delta t}^{n}, m \Delta t\right)-\varphi^{k}\left(r_{m \Delta t}^{n}, \lambda_{m \Delta t}^{n}, m \Delta t\right)\right|^{2}\right] } \\
\leq & C \frac{M N_{B} k}{N}+C \Delta t+C \sum_{l=1}^{k} \sum_{m=0}^{M}\left\{\operatorname{PrErr}^{l}(r, \lambda, m \Delta t)+\operatorname{AvErr}^{l}(r, \lambda, m \Delta t)\right\} \\
& +C \frac{1}{\Delta t^{2}} N_{B} \exp \left(\frac{-C N \Delta t^{3}}{N_{B}}\right) \exp \left(C N_{B} \ln \left(\frac{\sqrt{N_{B}}}{\Delta t^{3 / 2}}\right)\right) .
\end{aligned}
$$

The above proposition shows that the projection and averaging errors, PrErr and AvErr of (4.17), add up in time and number of contracts, which is the best that can be expected in general. Theorem 4.1 also highlights the tradeoff between minimizing PrErr and AvErr, which depend on the number of basis functions $N_{B}$ and number of averaging paths $N_{2}$, and algorithm speed in terms of the number of simulated paths $N$. In order to have

$$
\sum_{m=0}^{M}\left\{\operatorname{PrErr} r^{k}(r, \lambda, m \Delta t)+A v E r r^{k}(\lambda, m \Delta t)\right\}<\epsilon,
$$

for some fixed error $\epsilon$, we roughly need $N_{B} \sim \Delta t^{-2}$ (due to our two-dimensional state space $(r, \lambda))$ and $N_{2} \sim \Delta t^{-1}$. In turn, (4.18) implies that with this choice, to achieve convergence we must take

$$
N>\frac{C}{(\Delta t)^{7}} \ln \left((\Delta t)^{-5 / 2}\right)
$$


The algorithm complexity is $O\left(N \cdot N_{2} \cdot N_{B}^{3} \cdot M\right)$.

The main difficulty with the projection method is selecting good basis functions $\left\{B_{j}\right\}$. In practice, the choice is heuristic and seems to have a strong effect on empirical accuracy of results. Accordingly, some degree of customization or "black art" is necessary to perfect the method. To sidestep this problem there exist alternatives that avoid projection altogether, albeit at the cost of introducing other errors or increasing the computation time. Let us mention the use of kernel regression for computing conditional expectations (Carrière; 1996), as well as optimal quantization (Pagès et al.; 2005) and Markov chain approximation (Kushner and Dupuis; 2001). Overall, the projection method offers a good compromise between robustness, simple implementation and tractability.

\subsection{Numerical Example}

To illustrate our numerical algorithm, as well as the theoretical results of Section 3, we consider the following example. Let

$$
\left\{\begin{array}{l}
d r_{t}=\kappa_{r}\left(\bar{r}-r_{t}\right) d t+\sigma_{r} d B_{t} \\
d \lambda_{t}=\left(g+\frac{1}{2} \sigma_{\lambda}^{2}+\kappa_{\lambda}\left(g t+\ln \bar{\lambda}-\ln \lambda_{t}\right)\right) \lambda_{t} d t+\sigma_{\lambda} \lambda_{t} d B_{t}^{\lambda}
\end{array}\right.
$$

The model (4.20) corresponds to the short interest rate following a Vasicek model with mean-reversion level $\bar{r}$ and the hazard rate following the mean-reverting Brownian Gompertz model proposed in Milevsky and Promislow (2001) with Gompertz parameter $g$. This means that $\ln \lambda_{t}$ follows an Ornstein-Uhlenbeck model with linear drift $g$.

We allow a correlation $\rho=d\left\langle B, B^{\lambda}\right\rangle_{t}$ between the two Brownian motions $\left(B, B^{\lambda}\right)$. In discrete time after using the explicit Gaussian transition probability of OU process, (4.20) becomes

$$
\begin{array}{r}
\lambda_{t+\Delta t} \simeq \exp \left(\left(1-\mathrm{e}^{-\kappa_{\lambda} \Delta t}\right)(\ln \bar{\lambda}+g t)+g \Delta t+\mathrm{e}^{-\kappa_{\lambda} \Delta t} \ln \lambda_{t}\right. \\
\left.+\frac{\sigma_{\lambda} \sqrt{1-\mathrm{e}^{-2 \kappa_{\lambda} \Delta t}}}{\sqrt{2 \kappa_{\lambda}}}\left[\rho \Delta B_{t}+\sqrt{1-\rho^{2}} \epsilon_{t}\right]\right),
\end{array}
$$

where $\epsilon_{t} \sim \mathcal{N}(0,1)$ is a standard normal random variable, independent of $B$.

We implement (4.20) with $\bar{r}=0.06, \bar{\lambda}=0.05, \kappa_{r}=1, \kappa_{\lambda}=0.5, \sigma_{r}=0.02, \sigma_{\lambda}=$ $0.2, g=0.1$ (these are same parameter values used by Milevsky and Promislow (2001)) and using high risk-aversion $\gamma=0.3$. The horizon is taken to be $T=10$ years with an update of $\lambda$ every month (a total of 120 periods, $\Delta t=\frac{1}{12}$ ). In this context, we price the sale of up to twelve pure endowments to be payable at time $T$. Given the above parameters, we expect about $\mathbf{E}^{\lambda_{0}, 0}\left[\exp \left(-\int_{0}^{T} \lambda_{t} d t\right)\right] \approx 41.8 \%$ of individuals to survive to $T$. On the other 
hand, the 10 -year bond price is $F(0.06,0 ; 10)=0.5497$, so that according to Theorems 3.4-3.5,

$$
0.418 \cdot 0.5497 \cdot k \leq H^{(k)}(0.06,0.05,0) \leq 0.5497 \cdot k, \quad \forall k
$$

With these values, the difference between a pde solver implementing (2.29) and a Monte-Carlo algorithm implementing (4.10)-(4.11) is less than one percent, which is acceptable given the risk premiums computed below. This shows that even for stochastic hazard rates $\lambda$ with volatility that is several times larger than in real life, the piecewiseconstant assumption over medium-length time periods is quite good. The running times of the algorithms (in a straightforward and little optimized implementations in Matlab) are comparable and come in under one minute on a $2 \mathrm{GHz}$ desktop.

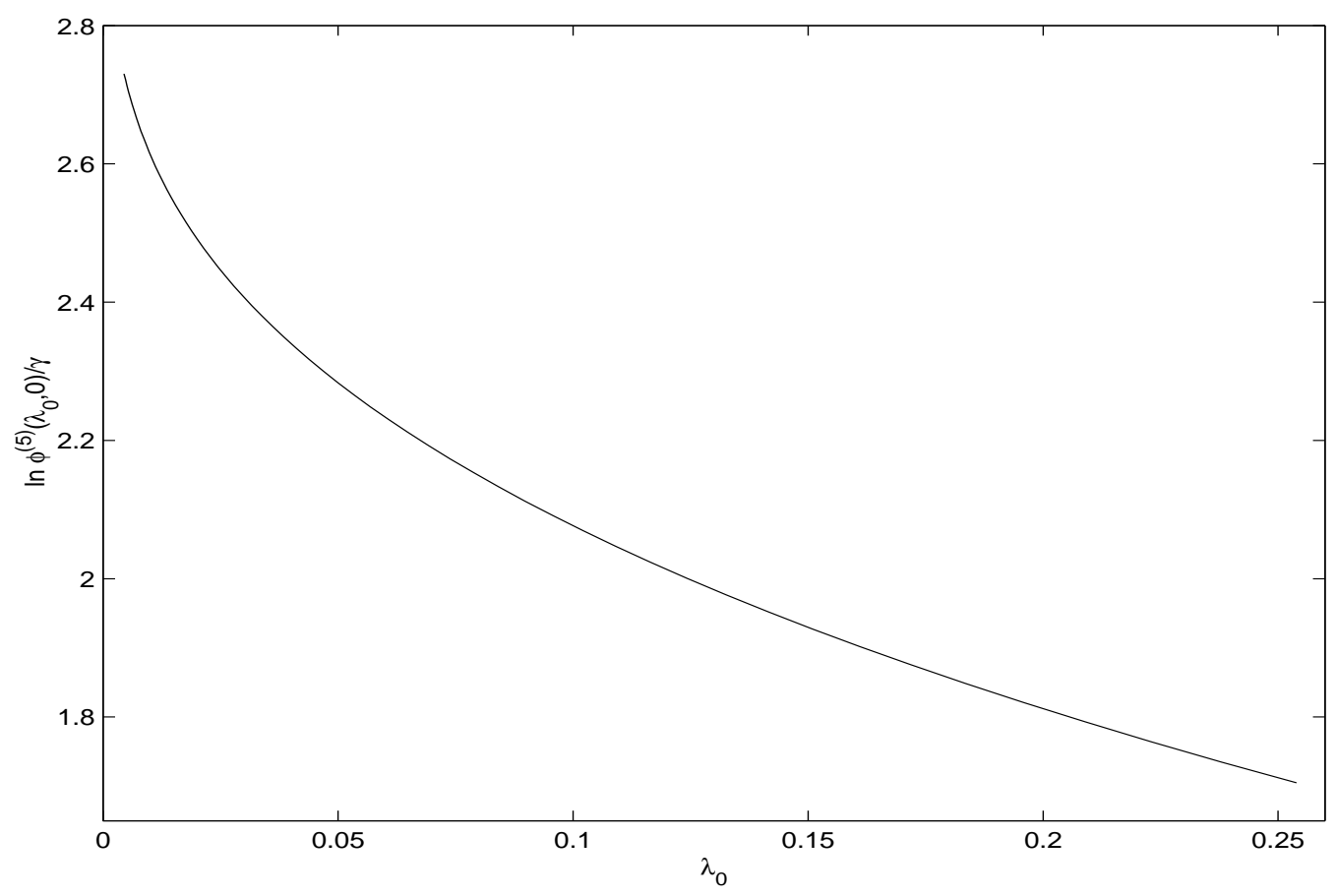

Figure 1: Graph of $\frac{1}{\gamma} \ln \phi^{(5)}\left(\lambda_{0}, 0\right)=\frac{H^{(5)}\left(0.06, \lambda_{0}, 0\right)}{F(0.06,0 ; 10)}$ as a function of initial mortality rate $\lambda_{0} ; \gamma=0.3$.

Figure 1 shows the relationship between $H^{(5)} / F$ and initial condition $\lambda_{0}$. As expected by Theorem 3.7, larger hazard rate decreases the indifference price; moreover $H$ is convex in $\lambda$. Figure 2 shows the relationship between $H^{(5)} /(5 F)$, the risk-aversion parameter $\gamma$ and time to expiration of contract. We see that for high $\gamma$ the indifference price is significantly above the "risk-neutral" price that corresponds to $\gamma=0$, with the premium being on the order of $30 \%$ for $\gamma=1$ and above parameter values. Also, the premium becomes more pronounced over longer time horizons as the stochasticity of $\lambda$ has more time to propagate 
through.

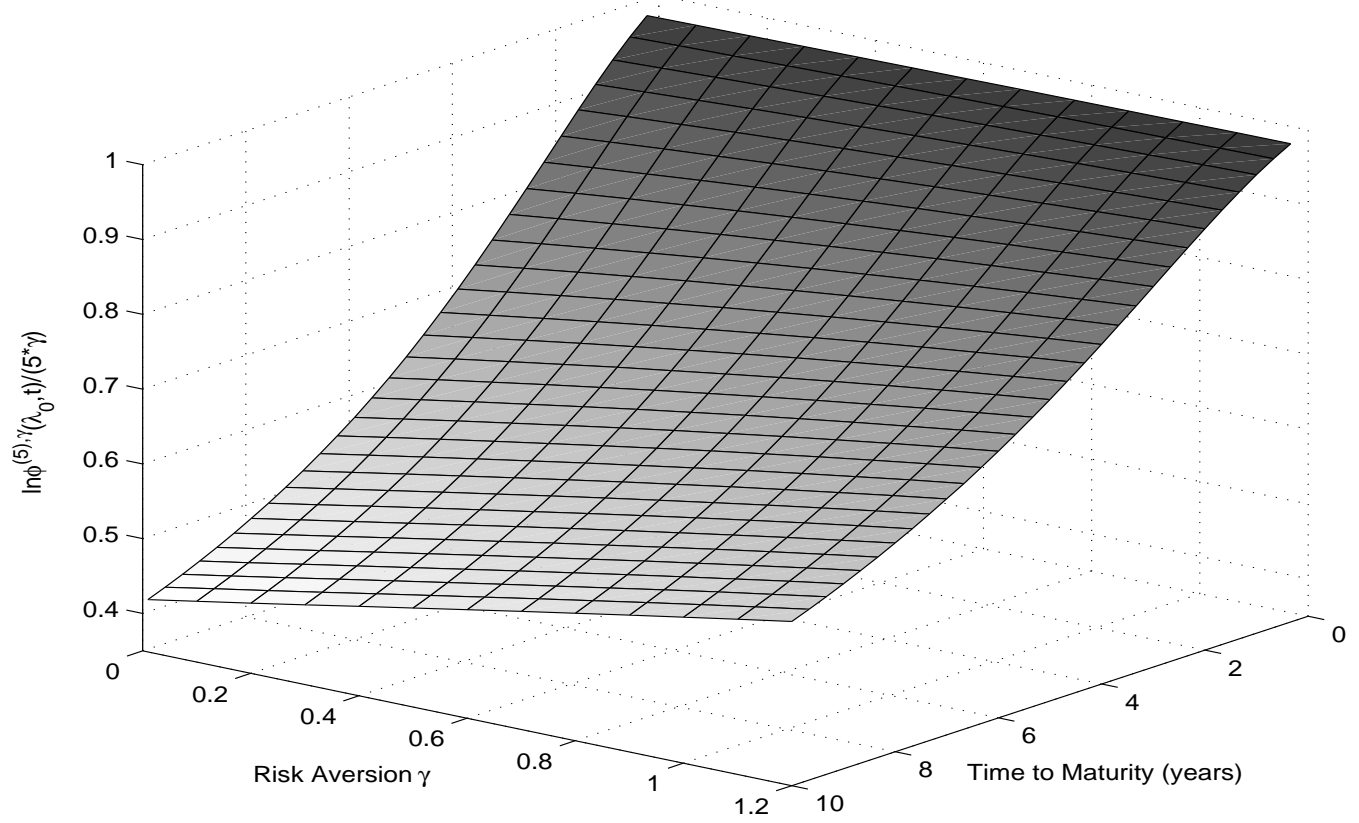

Figure 2: Graph of $H^{(5), \gamma}(r, \lambda, t) /(5 F(r, t ; 10))$ as a function of $\gamma$ and $t$, keeping $r=0.06, \lambda=0.05$ fixed. As proven in Theorem 3.8, $H$ is increasing in risk-aversion $\gamma$ and decreasing in time to maturity.

We also investigated the dependence of the indifference price on "secondary" parameters. Dependence on the volatility $\sigma_{\lambda}$ of the mortality rate is hard to measure because direct increase in $\sigma_{\lambda}$ lowers the probability of survival. Correcting for that, we find that $\sigma_{\lambda}$ has a weak but positive effect on the risk-premium $H^{(k), \gamma} / H^{(k), 0}$, see Table 1 below. This is consistent with our intuition about volatility of $\lambda$ being an additional "second-order" source of risk and the earlier Theorem 3.10. We can conclude that having stochastic hazard rates is not crucial when pricing single contracts. However, stochastic $\lambda$ becomes important when pricing several contracts due to the super-additivity property. We also found that the indifference price is quite insensitive to the correlation $\rho$ between $r$ and $\lambda$. Changing from no correlation $\rho=0$ to high positive correlation $\rho=0.99$ in this example increases the indifference price by about $2.5 \%$. Positive correlation between interest rates and mortality rates increases the overall volatility of payments that must be made and leads to larger risk.

\begin{tabular}{|lccccccc|}
\hline Volatility $\sigma_{\lambda}:$ & 0.1 & 0.2 & 0.3 & 0.4 & 0.5 & 0.7 & 0.9 \\
\hline Risk Premium: & $9.66 \%$ & $9.74 \%$ & $9.86 \%$ & $10.03 \%$ & $10.27 \%$ & $11.00 \%$ & $14.08 \%$ \\
\hline
\end{tabular}


Table 1: Risk premium as a function of volatility of hazard rate $\sigma_{\lambda}, \gamma=0.3$.

The price-per-risk is nearly constant in this example, as the following table demonstrates. Table 2 presents the incremental price-per-risk, $\frac{1}{\gamma}\left(\ln \phi^{(k)}\left(\lambda_{0}, t\right)-\ln \phi^{(k-1)}\left(\lambda_{0}, t\right)\right)$ with $\lambda_{0}=0.05, t=0$ as a function of number of contracts $k$. By Theorem 3.11 the priceper-risk increases, and as we see in a very slow linear fashion. This is because the hazard rate is not very volatile, so the induced correlation between mortality events is small. Thus, the upper bound of Theorem 3.4 is far from being tight. Unfortunately computation of $\phi^{(k)}$ for $k$ bigger than 20 is not numerically feasible, so that we do not know its behavior for a large number of contracts.

\begin{tabular}{|lcccccc|}
\hline No. of Contracts: & 1 & 2 & 3 & 4 & 8 & 12 \\
\hline Marginal Price: & 0.4557 & 0.4562 & 0.4567 & 0.4572 & 0.4592 & 0.4613 \\
\hline
\end{tabular}

Table 2: Marginal price-per-risk as a function of number of contracts.

\subsection{Comparison with Continuous-Time Model}

As already noted, the model of (4.1) can be seen as a discretized version of (2.1). Both admit solutions in terms of partial differential equations. For instance for pure endowments we have the pde (2.29), as well as a lattice-based Feynman-Kac solution of (4.11)-(4.12). It is instructive to compare them, as the two expressions sharpen our intuition about the difference between a continuous-time and discrete-time model.

To focus on the basic structure, let us consider the basic explicit finite differences method applied to these equations. The linear parabolic pde (2.29) is straighforward to discretize. In particular, taking a regular $(\lambda, t)$ grid with spacing $(\Delta \lambda, \Delta t)$ and writing $v_{n}^{m} \simeq \phi^{(k)}(n \Delta \lambda, m \Delta t), \bar{v}_{n}^{m}=\phi^{(k-1)}(n \Delta \lambda, m \Delta t)$ finite differencing gives

$$
\left\{\begin{aligned}
v_{n}^{m-1}= & v_{n}^{m}+\mu(n \Delta \lambda, m \Delta t) \cdot n \Delta \lambda \frac{\Delta t}{2 \Delta \lambda}\left(v_{n+1}^{m}-v_{n-1}^{m}\right) \\
& +\frac{1}{2} \sigma^{2}(m \Delta t) \cdot(n \Delta \lambda)^{2} \frac{\Delta t}{\Delta \lambda^{2}}\left(v_{n+1}^{m}-2 v_{n}^{m}+v_{n-1}^{m}\right)-k \cdot \Delta t(n \Delta \lambda)\left(v_{n}^{m}-\bar{v}_{n}^{m}\right), \\
v_{n}^{M}= & \mathrm{e}^{k \gamma}
\end{aligned}\right.
$$

plus appropriate boundary conditions. This can be re-written as

$$
v_{n}^{m-1}=v_{n}^{m}+p^{+} v_{n+1}^{m}+p^{0} v_{n}^{m}+p^{-} v_{n-1}^{m}-k \Delta t(n \Delta \lambda)\left(v_{n}^{m}-\bar{v}_{n}^{m}\right),
$$

where the weights $p^{+}, p^{0}, p^{-}$have the standard interpretation as transition probabilities of a discrete Markov chain $\tilde{\lambda}$ on the grid $(n \Delta \lambda, m \Delta t)$ whose conditional mean and variance are 
consistent with those of $\lambda$ (Kushner and Dupuis; 2001). The final answer is $H^{(k)}(n \Delta \lambda, 0)=$ $\frac{F(r, t ; T)}{\gamma} \ln v_{n}^{0}$ undoing the exponential terminal condition of (4.21).

On the other hand, if $r$ and $\lambda$ are independent, then (4.11)-(4.12) for pricing a pure endowment reduces to $H^{(k)}(\lambda, t)=F(r, t ; T) v^{k}(\lambda, t)$ where

$$
v^{k}(\lambda, t)=\frac{1}{\gamma} \ln \mathbf{E}^{\lambda, t}\left[\mathrm{e}^{\gamma v^{k}\left(\lambda_{t+\Delta t}, t+\Delta t\right)} \cdot\left(1-k \cdot \lambda_{t} \Delta t\right)+\mathrm{e}^{\gamma v^{k-1}\left(\lambda_{t+\Delta t}, t+\Delta t\right)} \cdot\left(k \cdot \lambda_{t} \Delta t\right)\right]
$$

and the Feynman-Kac formula followed by discretization using the same Markov chain $\tilde{\lambda}$ gives

$$
\left\{\begin{aligned}
v_{n}^{m-1}= & \frac{1}{\gamma} \ln \left((1-k(n \Delta \lambda) \Delta t)\left\{p^{+} \mathrm{e}^{\gamma v_{n+1}^{m}}+p^{0} \mathrm{e}^{\gamma v_{n}^{m}}+p^{-} \mathrm{e}^{\gamma v_{n-1}^{m}}\right\}\right. \\
& \left.+(k(n \Delta \lambda) \Delta t)\left\{p^{+} \mathrm{e}^{\gamma \bar{v}_{n+1}^{m}}+p^{0} \mathrm{e}^{\gamma \bar{v}_{n}^{m}}+p^{-} \mathrm{e}^{\gamma \bar{v}_{n-1}^{m}}\right\}\right), \\
v_{n}^{M}= & k
\end{aligned}\right.
$$

Because we have the same $\tilde{\lambda}$, the weights $p^{+}, p^{0}, p^{-}$are the same as in (4.22). We see that the nonlinearity appearing as an initial/terminal transformation in (4.21) is transformed into nonlinear local dynamics of (4.24). This is representative of the fact that the discrete time model can be seen as a series of continuous-time models with piecewise constant $\lambda$.

\section{Summary and Conclusions}

This paper has presented a novel treatment of pricing mortality contingent claims via indifference pricing. Our main objective was to demonstrate the feasibility and main features of using indifference valuation in a full-scale stochastic model driven by stochastic interest rate and stochastic hazard rate. As such we have necessarily faced a trade-off between realism and tractability. We strove chiefly for the second and accordingly focused on a simple case of risk-preferences combined with a selection of representative contracts.

Our model is not completely realistic; as with any actuarial model, a practitioner using our methodology would want to adjust and calibrate it to correspond to the products that her company is selling. To accommodate this, we have considered both continuous-time and discrete-time $\lambda$-dynamics and have outlined efficient numerical methods for each case. Having the latter also means we are not tied to a few special cases and can investigate various model extensions. For instance, one could study models that involve joint jumps in hazard and interest rates that might occur as a result of epidemia or war outbreaks. One could also analyze more complex insurance products, such as equity-linked annuities. This would require explicit modeling of investments in stocks represented by some price process 
$\left\{S_{t}\right\}$. However, the overall structure should remain the same and we conjecture that the general analysis of Section 2 will go through for the joint model involving $\left(S_{t}, r_{t}, \lambda_{t}\right)$.

Other approaches to indifference pricing are also possible. After this paper was complete we have learned of a closely related work by Dahl and Møller (2006) who study mean-variance indifference pricing in the same framework. It would be interesting to (numerically) compare the resulting hedging strategies and parameter dependencies.

\subsection{Relative Indifference Pricing}

The presented model can also be taken as a starting point for a more detailed study of hedging mortality claims. Indeed, in this paper we only analyzed the pricing question. However, the indifference pricing framework is also well-suited for risk-management. To be more specific, consider an insurer that has sold a claim $X$ and now wishes to hedge his exposure using some other claims $Y_{1}, Y_{2}, \ldots$. Then our framework provides a natural way of quantifying the hedging benefit of using $Y_{i}$ to offset $X$ via the resulting relative indifference price. Namely, start with $U^{X}$ as in (2.17) and define

$$
U^{X, Y_{i}}(w, r, \lambda, t)=\sup _{\pi \in \mathcal{A}} \mathbf{E}^{w, r, \lambda, t}\left[-\mathrm{e}^{-\gamma W_{T}^{X, Y_{i}}}\right]
$$

where $W^{X, Y_{i}}$ is a joint wealth process that includes payments coming out of both claims $X$ and $Y_{i}$. Then the relative indifference price of $Y_{i}$ given $X, H^{Y_{i} \mid X}$ solves

$$
U^{X, Y_{i}}\left(w+H^{Y_{i} \mid X}, r, \lambda, t\right)=U^{X}(w, r, \lambda, t) .
$$

Hence, $H^{Y_{i} \mid X}$ measures the benefit of selling $Y_{i}$ given that $X$ has been already sold. An example would be static hedging of annuities or pure endowments by life insurance contracts. The relative indifference price would be able to provide a risk-adjusted value of such a hedge, taking into account the stochastic nature of all the variables. Once this is done, one can select an optimal hedge, by minimizing some risk objective over the available hedging instruments $Y_{i}$. On a practical level, this would provide a guideline regarding the optimal annuity/life insurance ratio that the company should have. In general, such framework would lead to a consistent way of securitizing mortality risk, as envisioned by Cairns et al. (2004).

\subsection{Term Structure of Mortality}

A different interesting extension to consider is a possibility of modeling a heterogeneous population with multiple hazard rates $\lambda_{i}$. This would lead to a more realistic model 
that can take into effect different ages/characteristics of the individuals insured. Mathematically, this would mean replacing a scalar $\lambda$ by a vector of hazard rates. These hazard rates would correspond to mortality of different age groups and consequently are expected to be correlated, forming a term-structure of mortality, similar to a description given by Schrager (2006). Again, the relative indifference price described above would be a good tool for analyzing the effect of heterogeneous population on contract prices and respective hedging strategies.

The discrete-time model of Section 4 would be particularly beneficial for this setting, as the probabilistic numerical scheme described is easily extendable (and has good scaling properties) to higher dimensions, in contrast to pde methods that suffer from the curse of dimensionality. We hope to explore this possibility in future work, providing a first example of a tractable stochastic model with mortality age-structure.

\section{Acknowledgments}

We are deeply grateful to an anonymous referee for his careful reading of the paper and many suggestions that have significantly improved our presentation. We also thank Erhan Bayraktar, Masahiko Egami and other seminar participants at University of Michigan for their feedback on a preliminary version of this paper.

\section{References}

Becherer, D. (2003), Rational hedging and valuation of integrated risks under constant absolute risk aversion, Insurance: Mathematics and Economics 33(1): 1-28.

Bielecki, T., and M. Jeanblanc (2004), Indifference pricing of defaultable claims, Technical Report, Illinois Institute of Technology.

Biffis, E. (2005), Affine processes for dynamic mortality and actuarial valuation, Insurance: Mathematics and Economics, to appear.

Björk, T. (1998), Arbitrage Theory in Continuous Time, Oxford University Press, Oxford.

Blanchet-Scalliet, C., N. El Karoui, and L. Martellini (2005), Dynamic asset pricing theory with uncertain time-horizon, Journal of Economic Dynamics and Control, 29: 17371764.

Bouchard B., and N. Touzi (2004), Discrete-time approximation and Monte-Carlo simulation of backward stochastic differential equations, Stochastic Processes and Applications, 111(2): 175-206.

Cairns, A. J. G., D. Blake, and K. Dowd (2004), Pricing framework for securitization of mortality risk, Working paper, Heriot-Watt University. 
Carmona, R. and M. Ludkovski (2005), Optimal switching with applications to energy tolling agreements, Technical report, Princeton University.

Carrière, J. F. (1996), Valuation of the early-exercise price for options using simulations and nonparametric regression, Insurance: Mathematics and Economics, 19 (1): 19-30.

Dahl, M. (2004), Stochastic mortality in life insurance: Market reserves and mortalitylinked insurance contracts, Insurance: Mathematics and Economics, 35(1): 113-136.

Dahl, M. and T. Møller (2006), Valuation and hedging of life insurance liabilities with systematic mortality risk, Insurance: Mathematics and Economics, 39(2): 193-217.

Davis, M. H. A., V. G. Panas and T. Zarphipoulou (1993), European option pricing with transaction costs, SIAM Journal on Control and Optimization, 31: 470-493.

DiLorenzo, E. and M. Sibillo (2003), Longevity risk: Measurement and application perspectives, Working paper, Universita degli Studi di Napoli.

Gobet, E., J. Lemor and X. Warin (2006), Rate of convergence of an empirical regression method for solving generalized backward stochastic differential equations, Bernoulli, to appear.

Henderson, V. (2002), Valuation of claims on nontraded assets using utility maximization, Mathematical Finance, 12(4): 351-373.

Henderson, V. (2005), Valuing the option to invest in an incomplete market, Working paper, Princeton University.

Henderson, V., and D. Hobson (2005), Utility indifference pricing - an overview, in R. Carmona (ed.), Volume on Indifference Pricing, Princeton University Press, to appear.

Hodges, S. D. and A. Neuberger (1989), Optimal replication of contingent claims under transaction costs, Review of Futures Markets, 8: 222-239.

Karatzas, I. and S. E. Shreve (1991), Brownian Motion and Stochastic Calculus, second edition, Springer-Verlag, New York.

Kushner, H. J. and P. Dupuis (2001), Numerical Methods for Stochastic Control Problems in Continuous Time, second edition, Springer-Verlag, New York.

Lamberton, D. and B. Lapeyre (1996), Introduction to Stochastic Calculus Applied to Finance, Chapman \& Hall/CRC, Boca Raton, Florida.

Lee, R. D. and L. R. Carter (1992), Modeling and forecasting U.S. mortality, Journal of the American Statistical Association, 87 (419): 659-671.

Leland, H. E. (1985), Option pricing and replication with transactions costs, Journal of Finance, 40(5): 1283-1301. 
Longstaff F. and E. S. Schwartz (2001), Valuing American options by simulations: a simple least squares approach, The Review of Financial Studies, 14: 113-148.

Ludkovski, M. (2006), Financial hedging of operational flexibility, Working Paper, University of Michigan.

Milevsky, M. A. and S. D. Promislow (2001), Mortality derivatives and the option to annuitize, Insurance: Mathematics and Economics, 29(3): 299-318.

Milevsky, M. A., S. D. Promislow and V. R. Young (2005), Financial Valuation of mortality risk via the instantaneous Sharpe ratio, Working paper, University of Michigan.

Musiela, M. and T. Zariphopoulou (2004), A valuation algorithm for indifference prices in incomplete markets, Finance and Stochastics, 8 (3) : 399-414.

Olivieri, A. (2001), Uncertainty in mortality projections: An actuarial perspective, Insurance: Mathematics and Economics, 29(2): 231-245.

Pagès, G., H. Pham, and J. Printems (2004), Optimal quantization methods and applications to numerical problems in finance in Handbook of Computational and Numerical Methods in Finance, pages 253-297, Birkhäuser Boston.

Pratt, J. W. (1964), Risk aversion in the small and in the large, Econometrica, 32: 122-136.

Schrager, D.F. (2006), Affine stochastic mortality, Insurance: Mathematics and Economics, 38(1): 81-97.

Schweizer, M. (2001), A guided tour through quadratic hedging approaches, in E. Jouini, J. Cvitanic, M. Musiela (eds.), Option Pricing, Interest Rates and Risk Management, Cambridge University Press, 538-574.

Soininen, P. (1995), Stochastic variation of interest and mortality, Proceedings of the 5th AFIR International Colloquium, 871-904.

Walter, W. (1970), Differential and Integral Inequalities, Springer-Verlag, New York.

Young, V. R. and T. Zariphopoulou (2002), Pricing dynamic insurance risks using the principle of equivalent utility, Scandinavian Actuarial Journal, 4:246-279.

Young, V. R. (2003), Equity-indexed life insurance: Pricing and reserving using the principle of equivalent utility, North American Actuarial Journal, 7(1): 68-86.

Young, V. R. (2004), Pricing in an incomplete market with an affine term structure, Mathematical Finance, 14(3): 359-381. 